\title{
REGULARLY DISTRIBUTED SUBSETS IN THE COMPLEX PLANE
}

\author{
A. I. ABDULNAGIMOV AND A. S. KRIVOSHEYEV
}

\begin{abstract}
Certain conditions are studied under which there exists a regularly distributed set that is a part of a given sequence of complex numbers and, moreover, contains a given subsequence of that sequence. On this basis, splitting of entire functions and their asymptotic behavior are investigated. The results are also applied to problems concerning the completeness of systems of exponential monomials in convex domains and the representation of functions analytic on compact convex sets, as well as to the fundamental principle problem for invariant subspaces of functions.
\end{abstract}

\section{§1. INTRODUCTION}

Problems concerning the construction of regularly distributed sets (sets with angular density and regular sets) were considered by many authors. We mention the monographs [1, Chapter II.4] and [2, Chapter I.3, Items 1,2,4], as well as the papers [3, 4, 5, 6, 7]. The results were applied in order to construct entire functions with a given indicator, to study their asymptotic behavior and the possibility of their splitting, to investigate the problem of completeness for a system of exponential functions in the space of analytic functions, etc.

In this paper, we consider complex sequences of the first order. Note that, by using Theorem 1 in [4], our results obtained for such sequences can be extended to sequences of proximate order $\rho(r)$.

In $\S 2$, we obtain a condition equivalent to the following property: from a sequence $\Lambda^{2} \supseteq$ $\Lambda^{1}$ we can extract a measurable set $\Lambda$ that has a given angular density and contains $\Lambda^{1}$ (Theorem 2.4). In Theorem 2.9 and Lemma 2.10, we provide conditions under which, from a sequence $\Lambda^{2} \supseteq \Lambda^{1}$, we can extract a regularly distributed set $\Lambda$ that has a given angular density and contains $\Lambda^{1}$. These results contain most of the results mentioned above that concern the construction of regularly distributed sets.

In $\S \S 3$ and 4 , we consider various applications of the results of $\S 2$. In Theorems 3.3, 3.5, and 3.6, we study the splitting of functions of exponential type. Also, in Theorems 3.5 and 3.6 we obtain an asymptotic representation of an entire function with a measurable sequence of zeros. It generalizes the classical Levin representation of functions with regularly distributed zero set to the case of functions with measurable zero set. The representation in Theorem 3.5 is based on the representation of functions with zero set of zero density (see Lemma 3.2). A corollary to Lemma 3.2 refines the known result by M. Cartwright (see [8] and [2, I.1, Theorem 1.1.8]) about the type of a function with zero set of zero density. Another consequence of Lemma 3.2 yields a method for constructing entire functions of exponential type with a given indicator and with the minimal possible (zero) density.

2010 Mathematics Subject Classification. Primary 30D20.

Key words and phrases. Regularly distributed set, series of exponential functions, entire function, convex domain. 
In the last section, we consider problems concerning the representation of analytic functions as limits of sequences of exponential polynomials. In particular, in Theorem 4.3 we provide conditions under which functions analytic on the closure of a bounded convex domain can be represented by a series of exponential functions that converges uniformly on compact sets inside this domain. These conditions are based on Theorem 2.9 and on the known result by Leont'ev (see [2, IV.6, Theorem 4.6.4]). In contrast to Leont'ev's result, Theorem 4.3 is formulated solely in terms of geometrical characteristics of the domain and the exponents of the exponential functions involved. In Theorem 4.4, we use Theorem 2.9 and the results of [9] and [10] to obtain a criterion for the validity of the fundamental principle for invariant subspaces of analytic functions in a bounded convex domain. In contrast to [9] and [10, this criterion is also formulated solely in terms of the geometric characteristics of the domain and the spectrum of the differential operator in the invariant subspace in question.

\section{§2. Construction of Regularly distributed sets}

Let $\Lambda=\left\{\lambda_{k}, n_{k}\right\}_{k=1}^{\infty}$ be a sequence of pairwise distinct complex numbers $\lambda_{k} \neq 0$ and their multiplicities $n_{k}$. Let $B(z, r)$ be a disk of radius $r$ centered at a point $z$. By $n(r, \Lambda)$ we denote the number of points $\lambda_{k}$ (with their multiplicities) that fall into the disk $B(0, r), r>0$. The quantities

$$
\underline{n}(\Lambda)=\liminf _{r \rightarrow \infty} \frac{n(r, \Lambda)}{r}, \quad \bar{n}(\Lambda)=\limsup _{r \rightarrow \infty} \frac{n(r, \Lambda)}{r}
$$

are called the lower and the upper density of $\Lambda$, respectively. We say that the sequence $\Lambda$ has a density $n(\Lambda)$ (is measurable) if $\underline{n}(\Lambda)=\bar{n}(\Lambda)=n(\Lambda)<+\infty$. Suppose that the sequence $\widetilde{\Lambda}=\left\{\xi_{p}\right\}_{p=1}^{\infty},\left|\xi_{1}\right| \leq\left|\xi_{2}\right| \leq \ldots$, consists of the points $\lambda_{k}, k \geq 1$, and each point $\lambda_{k}$ occurs in $\widetilde{\Lambda}$ exactly $n_{k}$ times. Then

$$
\begin{aligned}
\limsup _{p \rightarrow \infty} \frac{p}{\left|\xi_{p}\right|} & \leq \lim _{p \rightarrow \infty} \frac{n\left(\left|\xi_{p}\right|+1, \tilde{\Lambda}\right)}{\left|\xi_{p}\right|} \\
& =\lim _{p \rightarrow \infty} \frac{n\left(\left|\xi_{p}\right|+1, \Lambda\right)}{\left|\xi_{p}\right|}=\lim _{p \rightarrow \infty} \frac{n\left(\left|\xi_{p}\right|+1, \Lambda\right)}{\left|\xi_{p}\right|+1}=n(\Lambda), \\
\liminf _{p \rightarrow \infty} \frac{p}{\left|\xi_{p}\right|} & \geq \lim _{p \rightarrow \infty} \frac{n\left(\left|\xi_{p}\right|-1, \tilde{\Lambda}\right)}{\left|\xi_{p}\right|} \\
& =\lim _{p \rightarrow \infty} \frac{n\left(\left|\xi_{p}\right|-1, \Lambda\right)}{\left|\xi_{p}\right|}=\lim _{p \rightarrow \infty} \frac{n\left(\left|\xi_{p}\right|-1, \Lambda\right)}{\left|\xi_{p}\right|-1}=n(\Lambda) .
\end{aligned}
$$

Thus, we have

$$
n(\Lambda)=n(\widetilde{\Lambda})=\lim _{p \rightarrow \infty} \frac{p}{\left|\xi_{p}\right|}
$$

We set

$$
\begin{aligned}
& \bar{n}_{0}(\Lambda, \delta)=\limsup _{r \rightarrow \infty} \frac{n(r, \Lambda)-n((1-\delta) r, \Lambda)}{\delta r}, \\
& \underline{n}_{0}(\Lambda, \delta)=\liminf _{r \rightarrow \infty} \frac{n(r, \Lambda)-n((1-\delta) r, \Lambda)}{\delta r} .
\end{aligned}
$$

The quantities

$$
\bar{n}_{0}(\Lambda)=\limsup _{\delta \rightarrow 0} \bar{n}_{0}(\Lambda, \delta), \quad \underline{n}_{0}(\Lambda)=\liminf _{\delta \rightarrow 0} \underline{n}_{0}(\Lambda, \delta)
$$

are called the minimal and the maximal density of $\Lambda$, respectively.

The following statements can be found [3, 4]. 
Lemma 2.1. Let $\Lambda=\left\{\lambda_{k}, n_{k}\right\}_{k=1}^{\infty}$.

1) If $\delta \in(0,1)$, then $\underline{n}_{0}(\Lambda) \leq \underline{n}_{0}(\Lambda, \delta) \leq \underline{n}(\Lambda) \leq \bar{n}(\Lambda)$ and $\bar{n}_{0}(\Lambda, \delta) \leq \bar{n}_{0}(\Lambda)$. If, moreover, $n(r, \Lambda)<+\infty, r>0$, then $\bar{n}(\Lambda) \leq \bar{n}_{0}(\Lambda, \delta)$;

2) If $\Lambda$ is measurable, then $\underline{n}_{0}(\Lambda)=\underline{n}_{0}(\Lambda, \delta)=n(\Lambda)=\bar{n}_{0}(\Lambda, \delta)=\bar{n}_{0}(\Lambda), \delta \in(0,1)$.

Proof. 1) Suppose $\bar{n}_{0}(\Lambda)=\tau<+\infty$ and $\delta \in(0,1)$. We fix $\varepsilon>0$. By the definition of the maximal density, we can find $\delta_{0} \in(0,1)$ such that $\bar{n}_{0}\left(\Lambda, \delta^{\prime}\right) \leq \tau+\varepsilon, \delta^{\prime} \in\left(0, \delta_{0}\right)$. Choose a natural number $p$ satisfying $\delta /((1-\delta) p)<\delta_{0}$. We put $\alpha_{j}=(1-(j-1) \delta / p)$, $r_{j}=\alpha_{j} r, \delta_{j}=\delta /\left(\alpha_{j} p\right), j=1, \ldots, p$. Then $\delta_{j} \in\left(0, \delta_{0}\right), j=1, \ldots, p$, and we have

$$
\begin{aligned}
\bar{n}_{0}(\Lambda, \delta) & =\limsup _{r \rightarrow \infty}(\delta r)^{-1} \sum_{j=1}^{p}\left(n\left(\alpha_{j} r, \Lambda\right)-n\left(\left(\alpha_{j}-\delta / p\right) r, \Lambda\right)\right) \\
& \leq(\delta)^{-1} \sum_{j=1}^{p} \alpha_{j} \delta_{j} \limsup _{r_{j} \rightarrow \infty} \frac{n\left(r_{j}, \Lambda\right)-n\left(\left(1-\delta_{j}\right) r_{j}, \Lambda\right)}{\delta_{j} r_{j}} \\
& =\sum_{j=1}^{p} \frac{\bar{n}_{0}\left(\Lambda, \delta_{j}\right)}{p} \leq \tau+\varepsilon .
\end{aligned}
$$

Since $\varepsilon>0$ is arbitrary, this implies $\bar{n}_{0}(\Lambda, \delta) \leq \bar{n}_{0}(\Lambda), \delta \in(0,1)$. The inequality $\underline{n}_{0}(\Lambda) \leq \underline{n}_{0}(\Lambda, \delta)$ can be proved similarly.

Suppose $n(r, \Lambda)<+\infty, r>0, \delta \in(0,1)$, and $\bar{n}_{0}(\Lambda, \delta)<+\infty$. By the definition of $\bar{n}_{0}(\Lambda, \delta)$, we can choose $r_{0}>0$ such that $(n(r, \Lambda)-n((1-\delta) r, \Lambda)) /(\delta r) \leq \bar{n}_{0}(\Lambda, \delta)+1$ for $r \geq r_{0}$. Suppose $r>r_{0}$ and $p(r)$ is the smallest natural number satisfying $r_{0} /(1-\delta)^{p(r)} \geq$ $r$. Then $r \geq r_{0} /(1-\delta)^{p(r)-1}$, and putting $r_{j}=r_{0} /(1-\delta)^{j}, j=0, \ldots, p(r)$, we obtain

$$
\begin{aligned}
\bar{n}(\Lambda) & \leq \limsup _{r \rightarrow \infty}\left(\sum_{j=0}^{p(r)} \frac{n\left(r_{0} /(1-\delta)^{j}, \Lambda\right)-n\left(r_{0} /(1-\delta)^{j-1}, \Lambda\right)}{r}+\frac{n\left((1-\delta) r_{0}, \Lambda\right)}{r}\right) \\
& \leq r_{0} \delta \limsup _{r \rightarrow \infty} \sum_{j=0}^{p(r)} \frac{n\left(r_{j}, \Lambda\right)-n\left((1-\delta) r_{j}, \Lambda\right)}{\delta r_{j}(1-\delta)^{j} r} \\
& \leq\left(\bar{n}_{0}(\Lambda, \delta)+1\right) r_{0} \delta \limsup _{r \rightarrow \infty} \sum_{j=0}^{p(r)} \frac{1}{(1-\delta)^{j} r} \\
& \leq\left(\bar{n}_{0}(\Lambda, \delta)+1\right) \delta \sum_{j=0}^{p(r)} \frac{(1-\delta)^{p(r)-1}}{(1-\delta)^{j}} \leq \frac{\left(\bar{n}_{0}(\Lambda, \delta)+1\right)}{1-\delta} .
\end{aligned}
$$

Therefore, $\bar{n}(\Lambda)<+\infty$. Consequently,

$\bar{n}(\Lambda) \leq \delta \limsup _{r \rightarrow \infty} \frac{n(r, \Lambda)-n((1-\delta) r, \Lambda)}{\delta r}+\limsup _{r \rightarrow \infty} \frac{n((1-\delta) r, \Lambda)}{r}=\delta \bar{n}_{0}(\Lambda, \delta)+(1-\delta) \bar{n}(\Lambda)$.

Hence, $\bar{n}(\Lambda) \leq \bar{n}_{0}(\Lambda, \delta), \delta \in(0,1)$. If $\underline{n}(\Lambda)<+\infty$, then the inequality $\underline{n}_{0}(\Lambda, \delta) \leq \underline{n}(\Lambda)$ can be proved similarly. The estimate $\underline{n}(\Lambda) \leq \bar{n}(\Lambda)$ follows from the definition of these quantities.

2) Suppose $\Lambda$ is measurable and $\delta \in(0,1)$. Then

$\underline{n}_{0}(\Lambda, \delta)=\bar{n}_{0}(\Lambda, \delta)=\lim _{r \rightarrow \infty} \frac{n(r, \Lambda)}{\delta r}-\lim _{r \rightarrow \infty} \frac{n((1-\delta) r, \Lambda)}{\delta r}=\frac{n(\Lambda)}{\delta}-\frac{(1-\delta) n(\Lambda)}{\delta}=n(\Lambda)$.

The lemma is proved.

Suppose $\Lambda^{1}=\left\{\lambda_{k}^{1}, n_{k}\right\}_{k=1}^{\infty}$ and $\Lambda^{2}=\left\{\lambda_{j}^{2}, m_{j}\right\}_{j=1}^{\infty}$. We say that $\Lambda^{1}$ is a subsequence of $\Lambda^{2}$ (and write $\Lambda^{1} \subseteq \Lambda^{2}$ ) if there exists a collection of indices $j(k), k \geq 1$, such 
that $\lambda_{k}^{1}=\lambda_{j(k)}^{2}$ and $n_{k} \leq m_{j(k)}, k \geq 1$. In this case, by $\Lambda^{2} \backslash \Lambda^{1}$ we denote the sequence $\tilde{\Lambda}^{2} \subseteq \Lambda^{2}$ consisting of all pairs $\left\{\lambda_{j}^{2}, m_{j}\right\}$, where $j \neq j(k), k \geq 1$, and all pairs $\left\{\lambda_{j(k)}^{2}, m_{j(k)}-n_{k}\right\}$ with $n_{k}<m_{j(k)}$. The union of arbitrary two sequences $\Lambda^{1}$ and $\Lambda^{2}$ is defined similarly. The set $\Lambda^{1} \cup \Lambda^{2}$ consists of all points $\lambda_{k}^{1}, \lambda_{j}^{2}$ together with their multiplicities; for coinciding points the multiplicities are added.

The proof of the following proposition is based on the method contained in the proof of Lemma 5 in [11].

Lemma 2.2. Suppose $\Lambda^{1}=\left\{\lambda_{k}^{1}, n_{k}\right\}_{k=1}^{\infty}, \Lambda^{2}=\left\{\lambda_{j}^{2}, m_{j}\right\}_{j=1}^{\infty}, \Lambda^{1} \subseteq \Lambda^{2}, n\left(r, \Lambda^{1}\right)<+\infty$, $r>0$, and $\tau \in[0,+\infty)$. The following statements are equivalent:

1) $\underline{n}_{0}\left(\Lambda^{2}\right) \geq \tau, \bar{n}_{0}\left(\Lambda^{1}\right) \leq \tau$;

2) there exists a sequence $\Lambda$ with density $\tau$ such that $\Lambda^{1} \subseteq \Lambda \subseteq \Lambda^{2}$.

Proof. 1) $\Rightarrow 2$ ). Since the various densities considered above are not affected by the arguments of the elements of our sequences, we may assume that $\lambda_{k}^{1}, \lambda_{j}^{2}>0, k, j \geq 1$. Also, we may assume that $\tau>0$, because the case where $\tau=0$ is trivial.

Suppose $\alpha=1 / \tau$ and $\Lambda_{p}^{2}$ contains all pairs $\left\{\lambda_{j}^{2}, m_{j}\right\}$ such that $\lambda_{j}^{2} \in[(p-1) \alpha, p \alpha)$, $p \geq 1$. We construct collections $\Lambda_{p}^{1}, p \geq 1$, in a similar way. By $n_{p}^{1}$ and $n_{p}^{2}$ we denote the numbers of points (taking multiplicities into account) in the sets $\Lambda_{p}^{1}$ and $\Lambda_{p}^{2}$, respectively. We provide a sequence $\Lambda$ as the union $\Lambda=\bigcup_{p \geq 1} \Lambda_{p}$. Applying induction, we construct sets $\Lambda_{p}, p \geq 1$, so that the following is true: for each $p \geq 1$ we have $\Lambda_{p}^{1} \subseteq \Lambda_{p} \subseteq \Lambda_{p}^{2}$ and the total number $N_{p}$ of points (taking multiplicities into account) in the sets $\Lambda_{1}, \ldots, \Lambda_{p}$ satisfies the inequalities

$$
\min \left\{p, N_{p-1}+n_{p}^{2}\right\} \leq N_{p} \leq \max \left\{p, N_{p-1}+n_{p}^{1}\right\},
$$

where $N_{0}=0$. Let $p=1$. If $\Lambda_{1}^{2}$ is empty, then we set $\Lambda_{1}=\varnothing$. Let $\Lambda_{1}^{2} \neq \varnothing$. If $\Lambda_{1}^{1} \neq \varnothing$, then we set $\Lambda_{1}=\Lambda_{1}^{1}$. Otherwise, we choose a point $\lambda_{j}^{2} \in[0, \alpha)$ arbitrarily and put $\Lambda_{1}=\left\{\lambda_{j}^{2}\right\}$.

Suppose we have already built the required sets $\Lambda_{p}$ for all $p<m$. Now we define $\Lambda_{m}$. If $N_{m-1}+n_{m}^{2} \leq m$, then we take $\Lambda_{m}^{2}$ as $\Lambda_{m}$. Let $N_{m-1}+n_{m}^{2}>m$. If we also have $N_{m-1}+n_{m}^{1} \geq m$, then we put $\Lambda_{m}=\Lambda_{m}^{1}$. Finally, suppose $N_{m-1}+n_{m}^{1}<m$. Then we choose an arbitrary set $\widetilde{\Lambda}_{m}^{2} \subseteq \Lambda_{m}^{2} \backslash \Lambda_{m}^{1}$ such that for $\Lambda_{m}=\Lambda_{m}^{1} \cup \widetilde{\Lambda}_{m}^{2}$, we have $N_{m}=m$.

Therefore, we have defined $\Lambda_{p}, p \geq 1$. Obviously, relation (2.1) is fulfilled and we have $\Lambda_{p}^{1} \subset \Lambda_{p} \subset \Lambda_{p}^{2}$. The last inclusion implies that $\Lambda^{1} \subseteq \Lambda \subseteq \Lambda^{2}$. We note that the assumption of the lemma and (2.1) imply the inequality $n(r, \Lambda)<+\infty, r>0$. It remains to prove that $\Lambda$ has density $\tau$. Let $r_{j} \rightarrow \infty$ be a sequence that implements the upper limit in the definition of $\bar{n}(\Lambda)$, and let $p(j), j \geq 1$, be the smallest natural number such that $p(j) \alpha \geq r_{j}$. If $N_{m}>m$ for all $m \geq 1$, then, using the definitions of $N_{m}$ and $\Lambda$, relation (2.1), and Lemma 2.1, we get

$$
\bar{n}(\Lambda)=\lim _{r \rightarrow \infty} \frac{n\left(r_{j}, \Lambda\right)}{r_{j}} \leq \limsup _{j \rightarrow \infty} \frac{N_{p(j)}}{p(j) \alpha} \leq \limsup _{j \rightarrow \infty} \frac{n\left(p(j) \alpha, \Lambda^{1}\right)}{p(j) \alpha} \leq \bar{n}\left(\Lambda^{1}\right) \leq \bar{n}_{0}\left(\Lambda^{1}\right) \leq \tau .
$$

Otherwise, for each $j \geq j_{0}$ we choose the largest natural number $m(j)$ such that $m(j) \leq$ $p(j)$ and $N_{m(j)} \leq m(j)$. Passing to a subsequence, we may assume that $m(j) / p(j)$ converges to some number $\gamma \in[0,1]$. Then by $(2.1)$ we have

$$
\begin{aligned}
\bar{n}(\Lambda) & \leq \limsup _{j \rightarrow \infty} \frac{N_{p(j)}}{p(j) \alpha} \leq \limsup _{j \rightarrow \infty} \frac{m(j)}{p(j) \alpha}+\limsup _{j \rightarrow \infty} \frac{N_{p(j)}-N_{m(j)}}{p(j) \alpha} \\
& \leq \frac{\gamma}{\alpha}+\limsup _{j \rightarrow \infty} \frac{n\left(p(j) \alpha, \Lambda^{1}\right)-n\left(m(j) \alpha, \Lambda^{1}\right)}{p(j) \alpha} .
\end{aligned}
$$


If $\gamma=0$, then $\bar{n}(\Lambda) \leq \lim \sup _{j \rightarrow \infty} n\left(p(j) \alpha, \Lambda^{1}\right) / p(j) \alpha \leq \tau$. Let $\gamma>0$. We choose $\delta^{\prime}>0$ such that $\gamma-\delta^{\prime}>0$ and set $\delta=1-\gamma+\delta^{\prime}$. Then $\delta \in(0,1)$ and $m(j)>(1-\delta) p(j)$, $j \geq j_{1}$. Therefore, Lemma 2.1 yields

$$
\bar{n}(\Lambda) \leq \frac{\gamma}{\alpha}+\limsup _{j \rightarrow \infty} \frac{n\left(p(j) \alpha, \Lambda^{1}\right)-n\left((1-\delta) p(j) \alpha, \Lambda^{1}\right)}{p(j) \alpha} \leq \tau \gamma+\delta \bar{n}_{0}(\Lambda, \delta) \leq \tau+\delta^{\prime} \tau .
$$

Since $\delta^{\prime}>0$ is arbitrary, we have $\bar{n}(\Lambda) \leq \tau$. The estimate $\underline{n}(\Lambda) \geq \tau$ can be proved similarly with the help of the left inequality in (2.1).

$2) \Rightarrow 1$ ). If 2) is fulfilled, then statement 1) follows immediately from Lemma 2.1 and the definition of $\underline{n}_{0}\left(\Lambda^{2}\right)$, and $\bar{n}_{0}\left(\Lambda^{1}\right)$. The lemma is proved.

A special case of Lemma 2.2 is Polya's theorem [3, Theorem 7] on a measurable kernel and a measurable hull, as well as its generalization [4, Theorem 3].

Corollary. Suppose $\widetilde{\Lambda}=\left\{\lambda_{k}, n_{k}\right\}_{k=1}^{\infty}, n(r, \widetilde{\Lambda})<+\infty, r>0, \bar{n}_{0}(\widetilde{\Lambda}) \leq \tau_{2} \in[0, \infty)$, and $\underline{n}_{0}(\widetilde{\Lambda}) \geq \tau_{1} \in[0, \infty)$. Then there exists a sequence $\widetilde{\Lambda}^{1} \subseteq \widetilde{\Lambda}$ with density $\tau_{1}$ and a sequence $\widetilde{\Lambda}^{2} \supseteq \widetilde{\Lambda}$ with density $\tau_{2}$.

Proof. The first statement follows immediately from Lemma 2.2 if we put $\Lambda^{2}=\widetilde{\Lambda}$ and $\Lambda^{1}=\varnothing$. In order to prove the second statement for $\tau_{2}>0$ (for $\tau_{2}=0$ it is trivial), we define an auxiliary sequence: $\Lambda^{\prime}=\{p \alpha\}_{p=1}^{\infty}, \alpha=1 / \tau_{2}$. We put $\Lambda^{1}=\widetilde{\Lambda}, \Lambda^{2}=\widetilde{\Lambda} \cup \Lambda^{\prime}$ and apply Lemma 2.2. The corollary is proved.

Now we consider some finer characteristics of a sequence $\Lambda=\left\{\lambda_{k}, n_{k}\right\}_{k=1}^{\infty}$. Suppose $\varphi_{1}, \varphi_{2} \in[-2 \pi, 2 \pi), \varphi_{2}-\varphi_{1} \in(0,2 \pi]$. We say that such values $\varphi_{1}, \varphi_{2}$ are admissible. We set

$$
\Gamma\left(\varphi_{1}, \varphi_{2}\right)\left(\Gamma\left(\varphi_{1}, \varphi_{2}\right], \Gamma\left[\varphi_{1}, \varphi_{2}\right]\right)=\left\{\lambda=t e^{i \varphi}: \varphi \in\left(\varphi_{1}, \varphi_{2}\right)\left(\left(\varphi_{1}, \varphi_{2}\right],\left[\varphi_{1}, \varphi_{2}\right]\right), t>0\right\} .
$$

Let $\Lambda\left(\varphi_{1}, \varphi_{2}\right)\left(\Lambda\left(\varphi_{1}, \varphi_{2}\right], \Lambda\left[\varphi_{1}, \varphi_{2}\right]\right)$ denote the sequence consisting of all pairs $\left\{\lambda_{k}, n_{k}\right\}$ such that $\lambda_{k} \in \Gamma\left(\varphi_{1}, \varphi_{2}\right)\left(\Gamma\left(\varphi_{1}, \varphi_{2}\right], \Gamma\left[\varphi_{1}, \varphi_{2}\right]\right)$.

The lower, upper, minimal, and maximal densities of $\Lambda$ in an angle $\Gamma\left(\varphi_{1}, \varphi_{2}\right)$ are the corresponding densities of the sequence $\Lambda\left(\varphi_{1}, \varphi_{2}\right)$. We note that the notion of the maximal density in an angle differs from the notion of the maximal angular density introduced in 12 .

We say (see [1, Chapter II.1]) that $\Lambda$ has an angular density $n\left(\Lambda, \varphi_{1}, \varphi_{2}\right)<+\infty$ (for the order one) if for all admissible $\varphi_{1}, \varphi_{2}$ (except, possibly, those in a countable set $\Phi_{\Lambda}$ ) the following identity is fulfilled: $\underline{n}\left(\Lambda\left(\varphi_{1}, \varphi_{2}\right)\right)=\bar{n}\left(\Lambda\left(\varphi_{1}, \varphi_{2}\right)\right)=n\left(\Lambda, \varphi_{1}, \varphi_{2}\right)$. We have $\tilde{\varphi} \in \Phi_{\Lambda} \backslash\{-2 \pi\}$ if and only if $\inf _{\varphi>0} \bar{n}(\Lambda(\widetilde{\varphi}-\varphi, \widetilde{\varphi}+\varphi))>0$, where $\varphi$ is sufficiently small. The number $-2 \pi$ belongs or does not belong to $\Phi_{\Lambda}$ simultaneously with $\tilde{\varphi}=0$.

By $\Sigma$ we denote the class of functions $\omega(\varphi)$ that are monotone nondecreasing on $[-2 \pi, 2 \pi]$ and possess the following properties: $\omega(0)=0, \omega$ is left-continuous, $\omega(\varphi)=$ $\omega(\varphi-2 \pi)-\omega(-2 \pi), \varphi \in[0,2 \pi)$. By $\Phi(\omega)$ we denote the set of discontinuity points for $\omega$.

Suppose $\Lambda$ has an angular density. Then it determines a unique function $\omega_{\Lambda} \in \Sigma$ by the following rule: for $\varphi_{1}, \varphi_{2} \in(-2 \pi, 0) \backslash \Phi_{\Lambda}$, and $\varphi \in\left(\varphi_{1}, \varphi_{1}+2 \pi\right) \backslash \Phi_{\Lambda}$, we have

$$
\omega_{\Lambda}\left(\varphi_{1}\right)=-\lim _{\varphi_{2} \rightarrow 0} n\left(\Lambda, \varphi_{1}, \varphi_{2}\right), \quad \omega_{\Lambda}(\varphi)=n\left(\Lambda, \varphi_{1}, \varphi\right)+\omega_{\Lambda}\left(\varphi_{1}\right) .
$$

More precisely, $\omega_{\Lambda}$ extends uniquely up to a function of class $\Sigma$, and the extension does not depend on $\varphi_{1}$. It is easily seen that the sets $\Phi_{\Lambda}$ and $\Phi\left(\omega_{\Lambda}\right)$ coincide. The definition of $\omega_{\Lambda}$ implies that $n\left(\Lambda, \varphi_{1}, \varphi_{2}\right)=\omega_{\Lambda}\left(\varphi_{2}\right)-\omega_{\Lambda}\left(\varphi_{1}\right)$ for all admissible $\varphi_{1}, \varphi_{2} \notin \Phi_{\Lambda}=\Phi\left(\omega_{\Lambda}\right)$. Moreover, we have $n(\Lambda)=\omega_{\Lambda}(\varphi+2 \pi)-\omega_{\Lambda}(\varphi), \varphi \in[-2 \pi, 0)$. We say that a sequence $\Lambda$ has angular density $\omega \in \Sigma$ if it has an angular density and $\omega_{\Lambda}=\omega$. 
Lemma 2.3. Suppose $\omega \in \Sigma$ and $\Lambda$ are such that the sequence $\Lambda(\varphi, \psi]$ has a density and $n(\Lambda(\varphi, \psi])=\omega(\psi)-\omega(\varphi)$ for some $\varphi_{1} \in(-2 \pi, 0) \backslash \Phi(\omega)$ and all $\varphi, \psi \notin \Phi(\omega)$ satisfying $\varphi_{1} \leq \varphi<\psi \leq \varphi_{1}+2 \pi$. Then $\Lambda$ has angular density $\omega$.

Proof. Let $\widetilde{\varphi}_{1}, \widetilde{\varphi}_{2} \notin \Phi_{\Lambda}$ be admissible. Depending on the location of the points $\tilde{\varphi}_{1}, \tilde{\varphi}_{2}$ on the interval $[-2 \pi, 2 \pi]$, there are several possible situations. We discuss two of them. The other cases can be treated similarly.

1. $\widetilde{\varphi}_{2}=\varphi_{1}$. In this case, we have $\Lambda\left(\widetilde{\varphi}_{1}, \widetilde{\varphi}_{2}\right)=\Lambda\left(\widetilde{\varphi}_{1}+2 \pi, \varphi_{1}+2 \pi\right) \subseteq \Lambda\left(\widetilde{\varphi}_{1}+2 \pi, \varphi_{1}+2 \pi\right]$. By our assumption, $\left(\varphi_{1}+2 \pi\right) \notin \Phi(\omega)$. We show that $\left(\tilde{\varphi}_{1}+2 \pi\right) \notin \Phi(\omega)$. Since $\tilde{\varphi}_{1} \notin \Phi_{\Lambda}$, we have

$$
\inf _{\varphi>0} \bar{n}\left(\Lambda\left(\widetilde{\varphi}_{1}+2 \pi-\varphi, \widetilde{\varphi}_{1}+2 \pi+\varphi\right)\right)=\inf _{\varphi>0} \bar{n}\left(\Lambda\left(\widetilde{\varphi}_{1}-\varphi, \widetilde{\varphi}_{1}+\varphi\right)\right)=0 .
$$

Let $\psi_{l} \rightarrow 0$ be such that $\left(\widetilde{\varphi}_{1}+2 \pi+\psi_{l}\right) \notin \Phi(\omega), l \geq 1$. Recalling the assumption, we get

$$
\begin{aligned}
\omega\left(\widetilde{\varphi}_{1}+2 \pi+\psi_{l}\right)-\omega\left(\widetilde{\varphi}_{1}+2 \pi-\psi_{l}\right) & =n\left(\Lambda\left(\widetilde{\varphi}_{1}+2 \pi-\psi_{l}, \widetilde{\varphi}_{1}+2 \pi+\psi_{l}\right]\right) \\
& \leq \bar{n}\left(\Lambda\left(\widetilde{\varphi}_{1}+2 \pi-2 \psi_{l}, \widetilde{\varphi}_{1}+2 \pi+2 \psi_{l}\right)\right) \rightarrow 0, \quad l \rightarrow \infty .
\end{aligned}
$$

Since $\omega$ is monotone nondecreasing, this implies the desired result. Therefore, using the definition of the upper density, the assumption, and Lemma 2.1, we obtain

$$
\bar{n}\left(\Lambda\left(\widetilde{\varphi}_{1}, \widetilde{\varphi}_{2}\right)\right) \leq \bar{n}\left(\Lambda\left(\widetilde{\varphi}_{1}+2 \pi, \widetilde{\varphi}_{2}+2 \pi\right]\right)=\omega\left(\varphi_{1}+2 \pi\right)-\omega\left(\widetilde{\varphi}_{1}+2 \pi\right) .
$$

On the other hand, similar considerations yield the inequality

$$
\begin{aligned}
\underline{n}\left(\Lambda\left(\tilde{\varphi}_{1}, \tilde{\varphi}_{2}\right)\right) & \geq \underline{n}\left(\Lambda\left(\tilde{\varphi}_{1}+2 \pi, \varphi_{1}+2 \pi-\psi_{l}\right]\right) \\
& =\omega\left(\varphi_{1}+2 \pi-\psi_{l}\right)-\omega\left(\widetilde{\varphi}_{1}+2 \pi\right), \quad l \geq 1,
\end{aligned}
$$

where $0<\psi_{l} \rightarrow 0$ and $\left(\varphi_{1}+2 \pi-\psi_{l}\right) \notin \Phi(\omega)$. Hence, using the continuity of $\omega$ at the point $\varphi_{1}+2 \pi$ and the previous inequality, we see that $\Lambda\left(\widetilde{\varphi}_{1}, \widetilde{\varphi}_{2}\right)$ has the density $\omega\left(\varphi_{1}+2 \pi\right)-\omega\left(\widetilde{\varphi}_{1}+2 \pi\right)=\omega\left(\varphi_{1}\right)-\omega\left(\widetilde{\varphi}_{1}\right)=\omega\left(\widetilde{\varphi}_{2}\right)-\omega\left(\tilde{\varphi}_{1}\right)$.

2. $\tilde{\varphi}_{2}>\varphi_{1}, \tilde{\varphi}_{1}<\varphi_{1}$. As above, it can be shown that $\tilde{\varphi}_{2}, \tilde{\varphi}_{1}+2 \pi \notin \Phi(\omega)$ and $\Lambda\left(\varphi_{1}, \tilde{\varphi}_{2}\right)$ has the density $\omega\left(\tilde{\varphi}_{2}\right)-\omega\left(\varphi_{1}\right)$. Then, recalling the assumption, we have

$$
\begin{aligned}
n\left(\Lambda\left(\widetilde{\varphi}_{1}, \widetilde{\varphi}_{2}\right)\right) & =n\left(\Lambda\left(\widetilde{\varphi}_{1}, \varphi_{1}\right]\right)+n\left(\Lambda\left(\varphi_{1}, \widetilde{\varphi}_{2}\right)\right) \\
& =n\left(\Lambda\left(\widetilde{\varphi}_{1}+2 \pi, \varphi_{1}+2 \pi\right]\right)+n\left(\Lambda\left(\varphi_{1}, \widetilde{\varphi}_{2}\right)\right) \\
& =\omega\left(\varphi_{1}+2 \pi\right)-\omega\left(\widetilde{\varphi}_{1}+2 \pi\right)+\omega\left(\widetilde{\varphi}_{2}\right)-\omega\left(\varphi_{1}\right) \\
& =\omega\left(\widetilde{\varphi}_{2}\right)-\omega\left(\widetilde{\varphi}_{1}\right) .
\end{aligned}
$$

Due to the continuity of $\omega$ at the point $\varphi_{1}$, we see that $\varphi_{1} \notin \Phi_{\Lambda}$.

Therefore, $\Lambda$ has the angular density $n\left(\Lambda, \widetilde{\varphi}_{1}, \widetilde{\varphi}_{2}\right)=\omega\left(\widetilde{\varphi}_{2}\right)-\omega\left(\widetilde{\varphi}_{1}\right)$. It remains to prove that $\omega_{\Lambda}=\omega$. By the fact last proved, this immediately follows from the definition of $\omega_{\Lambda}$, the left continuity of the function $\omega$, and the identity $\omega(0)=0$. The lemma is proved.

Now we prove an analog of Lemma 2.2 for finer characteristics of $\Lambda$.

Theorem 2.4. Suppose $\Lambda^{1} \subseteq \Lambda^{2}, n\left(r, \Lambda^{1}\right)<+\infty, r>0$, and $\omega \in \Sigma$. The following statements are equivalent:

1) for every admissible $\varphi_{1}, \varphi_{2} \notin \Phi(\omega)$, we have

$$
\underline{n}_{0}\left(\Lambda^{2}\left(\varphi_{1}, \varphi_{2}\right)\right) \geq \omega\left(\varphi_{2}\right)-\omega\left(\varphi_{1}\right), \quad \bar{n}_{0}\left(\Lambda^{1}\left(\varphi_{1}, \varphi_{2}\right)\right) \leq \omega\left(\varphi_{2}\right)-\omega\left(\varphi_{1}\right) ;
$$

2) there exists $\Lambda$ with angular density $\omega$ such that $\Lambda^{1} \subseteq \Lambda \subseteq \Lambda^{2}$. 
Proof. The implication 2) $\Rightarrow 1$ ) follows from Lemma 2.1 and the definition of the quantities $\underline{n}_{0}\left(\Lambda^{2}\left(\varphi_{1}, \varphi_{2}\right)\right)$ and $\bar{n}_{0}\left(\Lambda^{1}\left(\varphi_{1}, \varphi_{2}\right)\right)$.

$1) \Rightarrow 2$ ). We prove this implication in two stages. First, we build a special collection of completions $\widetilde{\Lambda}^{j} \subseteq \Lambda^{2}, j \geq 1$, of the sequence $\Lambda^{1}$. At the second stage we "glue" the pieces $\widetilde{\Lambda}^{j}$ together into a new completion $\Lambda$ that has the desired angular density.

1. Let $\varphi_{1} \in(-2 \pi, 0) \backslash \Phi(\omega)$. For each $j=1,2, \ldots$ we fix a collection of numbers $\varphi_{s}^{j} \notin$ $\Phi(\omega), s=1, \ldots, s(j)$, such that $\varphi_{1}^{j}=\varphi_{1}, \varphi_{1}^{j}<\varphi_{2}^{j}<\cdots<\varphi_{s(j)}^{j}<\varphi_{1}+2 \pi=\varphi_{s(j)+1}^{j}$ and $\varphi_{s+1}^{j}-\varphi_{s}^{j}<1 / j, s=1, \ldots, s(j)$. We set $\Lambda_{s}^{1, j}=\Lambda^{1}\left(\varphi_{s}^{j}, \varphi_{s+1}^{j}\right]$, and $\Lambda_{s}^{2, j}=\Lambda^{2}\left(\varphi_{s}^{j}, \varphi_{s+1}^{j}\right]$, $s=1, \ldots, s(j)$. Let $j=1,2, \ldots, s=1, \ldots, s(j)$, and $\varphi_{s+1}^{j}<\varphi \notin \Phi(\omega)$. Using 1), we have

$$
\bar{n}_{0}\left(\Lambda_{s}^{1, j}\right) \leq \bar{n}_{0}\left(\Lambda^{1}\left(\varphi_{s}^{j}, \varphi\right)\right) \leq \omega(\varphi)-\omega\left(\varphi_{s}^{j}\right) .
$$

By the continuity of $\omega$ at the point $\varphi_{s+1}^{j}$, this implies the inequality $\bar{n}_{0}\left(\Lambda_{s}^{1, j}\right) \leq \omega\left(\varphi_{s+1}^{j}\right)-$ $\omega\left(\varphi_{s}^{j}\right)$. Similarly (by considering $\left.\varphi<\varphi_{s+1}^{j}\right)$, we obtain $\underline{n}_{0}\left(\Lambda_{s}^{2, j}\right) \geq \omega\left(\varphi_{s+1}^{j}\right)-\omega\left(\varphi_{s}^{j}\right)$. Then Lemma 2.2 implies the existence of a completion $\widetilde{\Lambda}_{s}^{j} \subseteq \Lambda^{2}\left(\varphi_{s}^{j}, \varphi_{s+1}^{j}\right]$ of the sequence $\Lambda^{1}\left(\varphi_{s}^{j}, \varphi_{s+1}^{j}\right]$ such that

$$
n\left(\widetilde{\Lambda}_{s}^{j}\right)=\omega\left(\varphi_{s+1}^{j}\right)-\omega\left(\varphi_{s}^{j}\right), \quad s=1, \ldots, s(j), \quad j \geq 1 .
$$

Now we set $\widetilde{\Lambda}^{j}=\bigcup_{s} \widetilde{\Lambda}_{s}^{j}, j \geq 1$. We have the embeddings $\Lambda^{1} \subseteq \widetilde{\Lambda}^{j} \subseteq \Lambda^{2}$.

2. Let $j \geq 1$. By $(2.2)$, it is possible to find $R_{j-1}>0$ that satisfies the condition

$$
\left|\frac{n\left(r, \tilde{\Lambda}_{s}^{j}\right)}{r}-\left(\omega\left(\varphi_{s+1}^{j}\right)-\omega\left(\varphi_{s}^{j}\right)\right)\right|<\frac{1}{j s(j)}, \quad s=1, \ldots, s(j), \quad r \geq R_{j-1} .
$$

We may assume that $R_{j+1} \geq 2 R_{j}$. Let $\widetilde{\Lambda}^{j, j}$ be the collection of all pairs in $\widetilde{\Lambda}^{j}$ the first elements of which lie in the annulus $\left\{\lambda \in \mathbb{C}: R_{j-1} \leq|\lambda|<R_{j}\right\}$. We set $\Lambda=\bigcup_{j \geq 1} \tilde{\Lambda}^{j, j}$. By construction, we have $\Lambda^{1} \subseteq \Lambda \subseteq \Lambda^{2}$. It remains to show that $\Lambda$ has the angular density $\omega$.

Fix $\varphi, \psi \notin \Phi(\omega)$ such that $\varphi_{1} \leq \varphi<\psi \leq \varphi_{1}+2 \pi$. We prove the identity $n(\Lambda(\varphi, \psi])=$ $\omega(\psi)-\omega(\varphi)$. Suppose that $r>0$ and a number $j(r)$ is such that $R_{j(r)-1}<r \leq R_{j(r)}$. By construction, for any $j_{0}$ and all $r>R_{j_{0}}$ we have

$$
\begin{aligned}
n(r, \Lambda(\varphi, \psi])=n\left(R_{j_{0}}, \Lambda(\varphi, \psi]\right) & +\sum_{j=j_{0}+1}^{j(r)-1}\left(n\left(R_{j}, \tilde{\Lambda}^{j}(\varphi, \psi]\right)-n\left(R_{j-1}, \tilde{\Lambda}^{j}(\varphi, \psi]\right)\right) \\
& +n\left(r, \widetilde{\Lambda}^{j(r)}(\varphi, \psi]\right)-n\left(R_{j(r)-1}, \tilde{\Lambda}^{j(r)}(\varphi, \psi]\right) .
\end{aligned}
$$

For each $j \geq j_{1}$, there are numbers $1 \leq i(j)<l(j) \leq s(j)$ ensuring the inclusions $\bigcup_{s=i(j)+1}^{l(j)-1} \Gamma\left(\varphi_{s}^{j}, \varphi_{s+1}^{j}\right] \subseteq \Gamma(\varphi, \psi] \subseteq \bigcup_{s=i(j)}^{l(j)} \Gamma\left(\varphi_{s}^{j}, \varphi_{s+1}^{j}\right]$. Using the definition of $\tilde{\Lambda}^{j}$, we obtain

$$
\begin{aligned}
\sum_{s=i(j)+1}^{l(j)-1}\left(n\left(r, \widetilde{\Lambda}_{s}^{j}\right)-n\left(\widetilde{r}, \widetilde{\Lambda}_{s}^{j}\right)\right) & \leq n\left(r, \widetilde{\Lambda}^{j}(\varphi, \psi]\right)-n\left(\widetilde{r}, \widetilde{\Lambda}^{j}(\varphi, \psi]\right) \\
& \leq \sum_{s=i(j)}^{l(j)}\left(n\left(r, \widetilde{\Lambda}_{s}^{j}\right)-n\left(\widetilde{r}, \tilde{\Lambda}_{s}^{j}\right)\right), \quad 0<\widetilde{r}<r .
\end{aligned}
$$

Suppose $1 \leq i<l \leq s(j)$ and $r>\tilde{r} \geq R_{j}$. By (2.3), we have

$$
\left|\sum_{s=i}^{l}\left(n\left(r, \tilde{\Lambda}_{s}^{j}\right)-n\left(\tilde{r}, \tilde{\Lambda}_{s}^{j}\right)\right)-(r-\widetilde{r}) \sum_{s=i}^{l}\left(\omega\left(\varphi_{s+1}^{j}\right)-\omega\left(\varphi_{s}^{j}\right)\right)\right| \leq \frac{2 r}{j} .
$$


Thus,

$$
\left|\sum_{s=i}^{l}\left(n\left(r, \tilde{\Lambda}_{s}^{j}\right)-n\left(\widetilde{r}, \tilde{\Lambda}_{s}^{j}\right)\right)-(r-\widetilde{r})\left(\omega\left(\varphi_{l}^{j}\right)-\omega\left(\varphi_{i}^{j}\right)\right)\right| \leq \frac{2 r}{j} .
$$

By (2.5), this implies

$$
\begin{aligned}
& (r-\widetilde{r})\left(\omega\left(\varphi_{l(j)-1}^{j}\right)-\omega\left(\varphi_{i(j)+1}^{j}\right)\right)-\frac{2 r}{j} \leq n\left(r, \tilde{\Lambda}^{j}(\varphi, \psi]\right)-n\left(\widetilde{r}, \tilde{\Lambda}^{j}(\varphi, \psi]\right) \\
& \leq(r-\widetilde{r})\left(\omega\left(\varphi_{l(j)}^{j}\right)-\omega\left(\varphi_{i(j)}^{j}\right)\right)+\frac{2 r}{j}, \quad j \geq j_{1}, \quad r>\widetilde{r} \geq R_{j} .
\end{aligned}
$$

Let $\varepsilon>0$. By the continuity of $\omega$ at the points $\psi$ and $\varphi$, there exists $\delta>0$ such that

$$
|\omega(\tilde{\psi})-\omega(\widetilde{\varphi})-(\omega(\psi)-\omega(\varphi))|<\varepsilon, \quad \text { for all } \tilde{\psi}, \tilde{\varphi}:|\tilde{\psi}-\psi|<\delta, \quad|\widetilde{\varphi}-\varphi|<\delta .
$$

Choose a number $j_{0} \geq \max \left\{j_{1}, 1 / \delta, 1 / \varepsilon\right\}$. Then by (2.4), (2.6), (2.7), and the inequality $R_{j+1} \geq 2 R_{j}$, we obtain

$$
\begin{aligned}
n(r, \Lambda(\varphi, \psi]) \leq n\left(R_{j_{0}}, \Lambda(\varphi, \psi]\right) & +\sum_{j=j_{0}+1}^{j(r)-1}\left(\left(R_{j}-R_{j-1}\right)\left(\omega\left(\varphi_{l(j)}^{j}\right)-\omega\left(\varphi_{i(j)}^{j}\right)\right)+\frac{2 R_{j}}{j}\right) \\
& +\left(r-R_{j(r)-1}\right)\left(\omega\left(\varphi_{l(j)^{j}}\right)-\omega\left(\varphi_{i(j)}^{j}\right)\right)+\frac{2 r}{j} \\
\leq n\left(R_{j_{0}}, \Lambda(\varphi, \psi]\right) & +\sum_{j=j_{0}+1}^{j(r)-1}\left(\left(R_{j}-R_{j-1}\right)(\omega(\psi)-\omega(\varphi)+\varepsilon)+\frac{2 R_{j}}{j}\right) \\
& +\left(r-R_{j(r)-1}\right)(\omega(\psi)-\omega(\varphi)+\varepsilon)+\frac{2 r}{j} \\
\leq n\left(R_{j_{0}}, \Lambda(\varphi, \psi]\right)+ & (\omega(\psi)-\omega(\varphi)+\varepsilon)\left(\sum_{j=j_{0}+1}^{j(r)-1}\left(R_{j}-R_{j-1}\right)+r-R_{j(r)-1}\right) \\
& +\sum_{j=j_{0}+1}^{j(r)-1} \frac{2 R_{j}}{j}+\frac{2 r}{j} \\
\leq n\left(R_{j_{0}}, \Lambda(\varphi, \psi]\right)+ & (\omega(\psi)-\omega(\varphi)+\varepsilon)\left(r-R_{j_{0}}\right)+6 \varepsilon r, \quad r>R_{j_{0} .} .
\end{aligned}
$$

Similarly, we get the lower estimate

$$
n(r, \Lambda(\varphi, \psi]) \geq n\left(R_{j_{0}}, \Lambda(\varphi, \psi]\right)+(\omega(\psi)-\omega(\varphi)-\varepsilon)\left(r-R_{j_{0}}\right)-6 \varepsilon r, \quad r>R_{j_{0}} .
$$

Since $\varepsilon>0$ is arbitrary, from the last two inequalities it follows that $n(\Lambda(\varphi, \psi])=$ $\omega(\psi)-\omega(\varphi)$. By Lemma 2.3, this implies that $\Lambda$ has angular density $\omega$. The theorem is proved.

Recall that a sequence $\Lambda=\left\{\lambda_{k}, n_{k}\right\}_{k=1}^{\infty}$ is called a regularly distributed set (see 1, Chapter II.1]) for the order one if it has an angular density and Lindelöf's condition is satisfied, i.e., the limit $\lim _{r \rightarrow \infty} \mathcal{N}(r, \Lambda)$ exists, where

$$
\mathcal{N}(r, \Lambda)=\sum_{\left|\lambda_{k}\right|<r} \frac{n_{k}}{\lambda_{k}} .
$$

The following statements provide an answer to the question as to how a sequence with angular density can be "converted" into a regularly distributed set.

We say that $\Lambda$ is a sequence of general form if there exist $\varphi_{1}, \varphi_{2}, \varphi_{3} \in[-\pi, \pi)$ such that $\varphi_{1}<\varphi_{2}<\varphi_{3}, \varphi_{2}-\varphi_{1}<\pi, \varphi_{3}-\varphi_{2}<\pi, \varphi_{1}+2 \pi-\varphi_{3}<\pi$, and

$$
\underline{n}_{0}\left(\Lambda\left(\varphi_{j}-\varphi, \varphi_{j}+\varphi\right)\right)>0, \quad j=1,2,3, \quad \varphi \in(0, \pi / 2) .
$$


Observe that the function occurring on the left in this inequality and depending on $\varphi$ is monotone nondecreasing. Thus, it suffices that the inequality be fulfilled for some sequence $\varphi=\psi_{j, p} \rightarrow 0$.

Lemma 2.5. Suppose $a>1, \Lambda=\left\{\lambda_{k}, n_{k}\right\}_{k=1}^{\infty}$, and $\mathbb{C} \ni \gamma_{m} \rightarrow 0$, as $m \rightarrow \infty$. Let $\Lambda$ be a sequence of general form. Then there exists a sequence $T \subset \Lambda$ with zero density such that

$$
\sum_{m=1}^{l} \gamma_{m}-\mathcal{N}\left(a^{l+1}, T\right) \rightarrow 0, \quad l \rightarrow \infty .
$$

Proof. For convenience, we introduce the sequence $\widetilde{\Lambda}=\left\{\xi_{i}\right\}$ consisting of the points $\lambda_{k}$, $k \geq 1$ : each $\lambda_{k}$ occurs in $\tilde{\Lambda}$ exactly $n_{k}$ times. Let $\varphi_{1}, \varphi_{2}, \varphi_{3}$ be the numbers from the definition of a sequence of general form. We set

$$
\varphi_{0}=4^{-1} \min \left\{\pi-\left(\varphi_{2}-\varphi_{1}\right) ; \pi-\left(\varphi_{3}-\varphi_{2}\right) ; \pi-\left(\varphi_{1}+2 \pi-\varphi_{3}\right)\right\}<\pi / 4 .
$$

We note an important property of the numbers $\varphi_{0}, \varphi_{1}, \varphi_{2}, \varphi_{3}$. For any straight line passing through the origin and for each of two half-planes generated by this line, there exists $j=1,2,3$ such that the angle $\Gamma_{j}=\Gamma\left(\varphi_{j}-2 \varphi_{0}, \varphi_{j}+2 \varphi_{0}\right)$ lies in that half-plane.

We define a set $T$ by $T=\bigcup_{m=1}^{\infty} T_{m}$, where

$$
T_{m}=\left\{t_{l}\right\}_{l=\widetilde{p}(m-1)+1}^{\widetilde{p}(m)}=\left\{\xi_{i(m, p)}\right\}_{p=1}^{p(m)}
$$

is a subset of $\tilde{\Lambda}$ that lies in the annulus $\mathcal{K}(m)=\left\{\xi: a^{m}<|\xi| \leq a^{m+1}\right\}$. The case where $T_{m}=\varnothing$ (i.e., $\left.p(m)=0, \tilde{p}(m)=\widetilde{p}(m-1)\right)$ for some $m$ is not excluded.

We set $\tilde{p}(0)=p(0)=0, \gamma_{0}(0)=0, \gamma_{m}(0)=\gamma_{m-1}(p(m-1))+\gamma_{m}, m \geq 1$,

$$
\gamma_{m}(p)=\gamma_{m}(0)-\sum_{\mu=\tilde{p}(m-1)+1}^{\tilde{p}(m-1)+p} \frac{1}{t_{\mu}}, \quad p=1, \ldots, p(m) .
$$

Suppose $\widetilde{\Gamma}_{j}=\Gamma\left(\varphi_{j}-\varphi_{0}, \varphi_{j}+\varphi_{0}\right), j=1,2,3, \Pi(\varphi)=\left\{\xi \in \mathbb{C}: \operatorname{Re}\left(\xi e^{i \varphi}\right\}>0, \varphi(m, p)\right.$ is the argument of the number $\gamma_{m}(p)$, and $j(m, p), m \neq 0$, is an index such that $\Gamma_{j(m, p)} \subset$ $\Pi(\varphi(m, p))$.

For each $\geq 1$ we choose a collection $T_{m}=\left\{t_{l}\right\}_{l=\widetilde{p}(m-1)+1}^{\widetilde{p}(m-1)+p(m)}$ such that:

1) $p(m)$ is the smallest nonnegative integer for which either we have $\left|\gamma_{m}(p(m))\right| \leq$ $\left(a^{m} \sin \varphi_{0}\right)^{-1}$, or the set $\mathcal{K}(m) \cap \Gamma_{j(m, p(m))}$ contains no points of the sequence $\widetilde{\Lambda} \backslash T_{m}$;

2 ) for each $p=1, \ldots, p(m)$, the number $t_{\widetilde{p}(m-1)+p}$ is an arbitrary element $\xi_{i(m, p)}$ belonging to $\tilde{\Lambda} \backslash T_{m, p-1}$ (where $T_{m, 0}=\varnothing$ and $T_{m, p-1}=\left\{\xi_{i(m, s)}\right\}_{s=1}^{p-1}, p>1$ ) and lying in the intersection $\mathcal{K}(m) \cap \widetilde{\Gamma}_{j(m, p-1)}$.

Thus, the set $T=\bigcup_{m=1}^{\infty} T_{m}$ has been defined. We find an upper bound for the numbers $p(m)>0$. First, we prove the inequality

$$
\left|\gamma_{m}(p)\right| \leq\left|\gamma_{m}(p-1)\right|-2^{-1} a^{-m-1} \sin \varphi_{0}, \quad p=1, \ldots, p(m) .
$$

By $(2.8)$, we have $\gamma_{m}(p)=\gamma_{m}(p-1)-\left(\xi_{i(m, p)}\right)^{-1}$. Then the cosine rule yields

$$
\left|\gamma_{m}(p)\right|^{2}=\left|\gamma_{m}(p-1)\right|^{2}+\left|\xi_{i(m, p)}\right|^{-2}-2\left|\gamma_{m}(p-1)\right|\left|\xi_{i(m, p)}\right|^{-1} \cos \alpha,
$$

where $\alpha$ is one of two angles between the vectors $\gamma_{m}(p-1)$ and $\left(\xi_{i(m, p)}\right)^{-1}$ that does not exceed $\pi / 2-\varphi_{0}$ (it exists because $\left.\xi_{i(m, p)} \in \widetilde{\Gamma}_{j(m, p-1)}\right)$. Since $\xi_{i(m, p)} \in \mathcal{K}(m)$ and by 1$)$ 
we have $\left|\gamma_{m}(p-1)\right|>\left(a^{m} \sin \varphi_{0}\right)^{-1}$, it follows that

$$
\begin{aligned}
& \left|\gamma_{m}(p-1)\right|^{2}-\left|\gamma_{m}(p)\right|^{2} \geq 2\left|\gamma_{m}(p-1)\right|\left|\xi_{i(m, p)}\right|^{-1} \sin \varphi_{0}-\left|\xi_{i(m, p)}\right|^{-2} \\
& \quad=\left|\gamma_{m}(p-1)\right|\left|\xi_{i(m, p)}\right|^{-1}\left(2 \sin \varphi_{0}-\left(\left|\xi_{i(m, p)}\right|\left|\gamma_{m}(p-1)\right|\right)^{-1}\right) \\
& \quad \geq\left|\gamma_{m}(p-1)\right|\left|\xi_{i(m, p)}\right|^{-1}\left(2 \sin \varphi_{0}-\sin \varphi_{0}\right) \geq\left|\gamma_{m}(p-1)\right| a^{-m-1} \sin \varphi_{0} .
\end{aligned}
$$

In particular, $\left|\gamma_{m}(p-1)\right|>\left|\gamma_{m}(p)\right|$. Therefore,

$$
\begin{aligned}
2 \mid \gamma_{m} & (p-1) \mid\left(\left|\gamma_{m}(p-1)\right|-\left|\gamma_{m}(p)\right|\right) \\
& \geq\left(\left|\gamma_{m}(p-1)\right|+\left|\gamma_{m}(p)\right|\right)\left(\left|\gamma_{m}(p-1)\right|-\left|\gamma_{m}(p)\right|\right) \\
& \geq\left|\gamma_{m}(p-1)\right| a^{-m-1} \sin \varphi_{0} .
\end{aligned}
$$

This implies inequality (2.9). By applying it $p(m)$ times, we get

$$
0 \leq\left|\gamma_{m}(p(m))\right| \leq\left|\gamma_{m}(0)\right|-2^{-1} a^{-m-1} p(m) \sin \varphi_{0}, \quad m \geq 1 .
$$

(For $p(m)=0$, the inequality is trivial.) Thus, we have

$$
p(m) \leq 2 a^{m+1}\left(\sin \varphi_{0}\right)^{-1}\left|\gamma_{m}(0)\right|, \quad m \geq 1 .
$$

Now we show that

$$
\gamma_{l}(p(l))=\sum_{m=1}^{l} \gamma_{m}-\mathcal{N}\left(a^{l+1}, T\right) \rightarrow 0, \quad l \rightarrow \infty .
$$

By assumption, there is $\tau>0$ such that

$$
\underline{n}_{0}\left(\widetilde{\Lambda}\left(\varphi_{j}-\varphi_{0}, \varphi_{j}+\varphi_{0}\right)\right) \geq 2 \tau a /(a-1), \quad j=1,2,3 .
$$

Then, taking Lemma 2.1 into account (wherein we put $\delta=1-a^{-1}$ ), we see that there exists $m_{0}$ with

$$
\begin{array}{r}
n\left(a^{m+1}, \widetilde{\Lambda}\left(\varphi_{j}-\varphi_{0}, \varphi_{j}+\varphi_{0}\right)\right)-n\left(a^{m}, \tilde{\Lambda}\left(\varphi_{j}-\varphi_{0}, \varphi_{j}+\varphi_{0}\right)\right) \geq \tau a^{m+1}, \\
j=1,2,3, \quad m \geq m_{0} .
\end{array}
$$

By (2.10) and 1), 2), this implies

$$
\left|\gamma_{m}(p(m))\right| \leq \max \left\{\left(a^{m} \sin \varphi_{0}\right)^{-1},\left|\gamma_{m}(0)\right|-2^{-1} \tau \sin \varphi_{0}\right\}, \quad m \geq m_{0} .
$$

The conditions of the lemma allow us to assume that

$$
\left|\gamma_{m}\right|+\left(a^{m-1} \sin \varphi_{0}\right)^{-1} \leq 4^{-1} \tau \sin \varphi_{0}, \quad m \geq m_{0}
$$

Suppose that $\left|\gamma_{m}(p(m))\right|>\left(a^{m} \sin \varphi_{0}\right)^{-1}$ for all $m \geq m_{0}$. Then by $(2.13),(2.14)$, and the definition of $\gamma_{m}(0)$, we have

$$
\begin{aligned}
\left|\gamma_{l}(p(l))\right| & \leq\left|\gamma_{l}(0)\right|-2^{-1} \tau \sin \varphi_{0} \leq\left|\gamma_{l-1}(p(l-1))\right|+\left|\gamma_{l}\right|-2^{-1} \tau \sin \varphi_{0} \\
& \left.\leq\left|\gamma_{l-1}(p(l-1))\right|-4^{-1} \tau \sin \varphi_{0} \leq \cdots \leq \mid \gamma_{m_{0}}\right)\left(p\left(m_{0}\right)\right) \mid-4^{-1} \tau\left(l-m_{0}\right) \sin \varphi_{0} .
\end{aligned}
$$

For large $l$, the left-hand side becomes negative, a contradiction. Therefore, there exists $m_{1} \geq m_{0}$ such that

$$
\left|\gamma_{m_{1}}\left(p\left(m_{1}\right)\right)\right| \leq\left(a^{m_{1}} \sin \varphi_{0}\right)^{-1}
$$

Then by (2.14) we obtain

$$
\left|\gamma_{m_{1}+1}(0)\right|-2^{-1} \tau \sin \varphi_{0} \leq\left|\gamma_{m_{1}}\left(p\left(m_{1}\right)\right)\right|+\left|\gamma_{m_{1}+1}\right|-2^{-1} \tau \sin \varphi_{0} \leq 0
$$

Therefore, by $(2.13)$ we have $\left|\gamma_{m_{1}+1}\left(p\left(m_{1}+1\right)\right)\right| \leq\left(a^{m_{1}+1} \sin \varphi_{0}\right)^{-1}$. This means that (2.12) is true. It remains to show that $T$ has zero density. Using (2.11) and (2.12), the assumption of the lemma, and the definition of $\gamma_{m}(0)$, we have

$$
\frac{p(m)}{a^{m}} \leq \frac{2 a\left|\gamma_{m}(0)\right|}{\sin \varphi_{0}} \leq \frac{2 a\left(\left|\gamma_{m-1}(p(m-1))\right|+\left|\gamma_{m}\right|\right)}{\sin \varphi_{0}} \rightarrow 0, \quad m \rightarrow \infty
$$


We fix $\varepsilon>0$. Then there exists a number $m(\varepsilon)$ such that $p(m) \leq \varepsilon a^{m}, m \geq m(\varepsilon)$. Suppose that $r>a^{m(\varepsilon)}$ and that $m(r)$ is chosen so that $a^{m(r)} \leq r<a^{m(r)+1}$. Then

$$
\begin{aligned}
\frac{n(r, T)}{r} & =\frac{n\left(a^{m(\varepsilon)}, T\right)}{r}+\frac{n\left(a^{m(r)+1}, T\right)-n\left(a^{m(\varepsilon)}, T\right)}{r} \\
& \leq \frac{n\left(a^{m(\varepsilon)}, T\right)}{r}+\frac{p(m(\varepsilon))+\cdots+p(m(r))}{a^{m(r)}} \leq \frac{n\left(a^{m(\varepsilon)}, T\right)}{r}+\varepsilon \frac{a^{m(\varepsilon)}+\cdots+a^{m(r)}}{a^{m(r)}} .
\end{aligned}
$$

This implies $\bar{n}(T) \leq \varepsilon a /(a-1)$. Since $\varepsilon>0$ is arbitrary, the lemma is proved.

Lemma 2.6. Suppose $\Lambda=\left\{\lambda_{k}, n_{k}\right\}_{k=1}^{\infty}$ has density $\tau \geq 0$. Suppose that a $>1, r_{2}>$ $r_{1}>0$, and $r_{2} / r_{1} \leq a$. Then we have the representation

$$
\begin{array}{r}
\sum_{r_{1} \leq\left|\lambda_{k}\right|<r_{2}} \frac{n_{k}}{\left|\lambda_{k}\right|}=\tau \ln \left(\frac{r_{2}}{r_{1}}\right)+\varepsilon\left(r_{1}, r_{2}\right), \quad \varepsilon\left(r_{1}, r_{2}\right) \rightarrow 0, \\
r_{1} \rightarrow \infty, \quad r_{2}>r_{1}>0, \quad r_{2} / r_{1} \leq a,
\end{array}
$$

(i.e., $\varepsilon\left(r_{1}, r_{2}\right) \rightarrow 0, r_{1} \rightarrow \infty$, uniformly in $r_{2}$ such that $\left.r_{2}>r_{1}>0, r_{2} / r_{1} \leq a\right)$.

Remark. If the annulus $r_{1} \leq|\lambda|<r_{2}$ contains no points $\lambda_{k}$, we set the left-hand side of this identity equal to zero.

Proof. As in the proof of Lemma 2.5, instead of $\Lambda$ we consider the sequence $\widetilde{\Lambda}=\left\{\xi_{p}\right\}$ constructed by $\Lambda$. We assume that $\left|\xi_{1}\right| \leq\left|\xi_{2}\right| \leq \ldots$ and $n(r, \widetilde{\Lambda}) \rightarrow+\infty, r \rightarrow \infty$ (otherwise, the claim of the lemma becomes trivial).

Let $\tau=0$. Then

$$
\sum_{r_{1} \leq\left|\lambda_{k}\right|<r_{2}} \frac{n_{k}}{\left|\lambda_{k}\right|} \leq \frac{1}{r_{1}} \sum_{r_{1} \leq\left|\lambda_{k}\right|<a r_{1}} n_{k}=\frac{1}{r_{1}}\left(n\left(a r_{1}, \Lambda\right)-n\left(r_{1}, \Lambda\right)\right) \rightarrow 0, \quad r_{1} \rightarrow \infty .
$$

Now, let $\tau>0$. By Euler's representation, we have

$$
\sum_{p=1}^{n} \frac{1}{p}=\ln n+\beta+\beta(n), \quad \beta(n) \rightarrow 0, \quad n \rightarrow \infty,
$$

where $\beta$ is the Euler constant. By assumption, $\widetilde{\Lambda}$ has density $\tau$, i.e., we have

$$
\begin{aligned}
\left|\xi_{p}\right| & =p /(\tau+\delta(p)), \quad \delta(p) \rightarrow 0, \quad p \rightarrow \infty, \\
n(r, \widetilde{\Lambda}) & =\tau r+\varepsilon(r) r, \quad \varepsilon(r) \rightarrow 0, \quad r \rightarrow \infty .
\end{aligned}
$$

By (2.15), this implies

$$
\begin{aligned}
& \sum_{r_{1} \leq\left|\lambda_{k}\right|<r_{2}} \frac{n_{k}}{\left|\lambda_{k}\right|}=\sum_{r_{1} \leq\left|\xi_{p}\right|<r_{2}} \frac{1}{\left|\xi_{p}\right|}=\sum_{r_{1} \leq\left|\xi_{p}\right|<r_{2}} \frac{\tau+\delta(p)}{p} \\
& =\tau \sum_{p=n\left(r_{1}, \widetilde{\Lambda}\right)+1}^{n\left(r_{2}, \widetilde{\Lambda}\right)} \frac{1}{p}+\sum_{p=n\left(r_{1}, \widetilde{\Lambda}\right)+1}^{n\left(r_{2}, \widetilde{\Lambda}\right)} \frac{\delta(p)}{p} \\
& =\tau \ln \frac{n\left(r_{2}, \widetilde{\Lambda}\right)}{n\left(r_{1}, \widetilde{\Lambda}\right)}+\tau\left(\beta\left(n\left(r_{2}, \widetilde{\Lambda}\right)\right)-\beta\left(n\left(r_{1}, \widetilde{\Lambda}\right)\right)\right)+\sum_{p=n\left(r_{1}, \widetilde{\Lambda}\right)+1}^{n\left(r_{2}, \widetilde{\Lambda}\right)} \frac{\delta(p)}{p} \\
& =\tau \ln \frac{r_{2}}{r_{1}}+\tau\left(\ln \frac{\tau+\varepsilon\left(r_{2}\right)}{\tau+\varepsilon\left(r_{1}\right)}+\beta\left(n\left(r_{2}, \widetilde{\Lambda}\right)\right)-\beta\left(n\left(r_{1}, \widetilde{\Lambda}\right)\right)\right)+\sum_{p=n\left(r_{1}, \widetilde{\Lambda}\right)+1}^{n} \frac{\delta(p)}{p} .
\end{aligned}
$$


We fix $\varepsilon>0$. In accordance with (2.15) and (2.16), we can choose a number $p_{0}$ such that $|\delta(p)| \leq \varepsilon,|\beta(n)| \leq \varepsilon, p, n \geq p_{0}$. Relation (2.16) allows us to choose $r(\varepsilon)>0$ such that

$$
\left|\ln \frac{\tau+\varepsilon\left(r_{2}\right)}{\tau+\varepsilon\left(r_{1}\right)}\right| \leq \varepsilon, \quad n\left(r_{1}, \widetilde{\Lambda}\right) \geq p_{0}, \quad r_{2}>r_{1}>r(\varepsilon) .
$$

Then

$$
\left|\varepsilon\left(r_{1}, r_{2}\right)\right| \leq 3 \varepsilon \tau+\varepsilon \sum_{p=n\left(r_{1}, \widetilde{\Lambda}\right)+1}^{n\left(a r_{1}, \widetilde{\Lambda}\right)} \frac{1}{p} \leq 3 \varepsilon \tau+\varepsilon(\ln a+3 \varepsilon), \quad r_{2}>r_{1}>r(\varepsilon), \quad r_{2} / r_{1} \leq a .
$$

The lemma is proved.

Let $K$ be a compact convex set. It uniquely determines a function of class $\Sigma$ via the arclength on its boundary $\partial K$. For each $\varphi \in \mathbb{R}$, let $L(\varphi, K)$ denote the intersection of the support line

$$
l(\varphi, K)=\left\{z: \operatorname{Re}\left(z e^{-i \varphi}\right)=H(\varphi, K)\right\}, \quad H(\varphi, K)=\sup _{z \in K} \operatorname{Re}\left(z e^{-i \varphi}\right),
$$

and the boundary $\partial K(H(\varphi, K)$ is the support function of the compact set $K)$. The set $L(\varphi, K)$ is either a point (we denote it by $z(\varphi, K)$ ), or a line segment. The set $\Psi(K)$ of directions $\varphi$ for which $L(\varphi, K)$ is a segment, is at most countable. Suppose that $\varphi_{1}, \varphi_{2} \notin \Psi(K), \varphi_{2}-\varphi_{1} \in(0,2 \pi)$, and $s\left(\varphi_{1}, \varphi_{2}, K\right)$ is the length of the arc of $\partial K$ that connects the points $z\left(\varphi_{1}, K\right)$ and $z\left(\varphi_{2}, K\right)$ : we move along it from $z\left(\varphi_{1}, K\right)$ to $z\left(\varphi_{2}, K\right)$ in the positive direction (counterclockwise). Let $\varphi_{1}, \varphi_{2} \in(-2 \pi, 0) \backslash \Psi(K)$, and let $\varphi \in\left(\varphi_{1}, \varphi_{1}+2 \pi\right) \backslash \Phi(K)$. The function

$$
\omega\left(\varphi_{1}, K\right)=-\lim _{\varphi_{2} \rightarrow 0} s\left(\varphi_{1}, \varphi_{2}, K\right), \quad \omega(\varphi, K)=s\left(\varphi_{1}, \varphi, K\right)+\omega\left(\varphi_{1}, K\right)
$$

can uniquely be extended to a function of class $\Sigma$, and the extension does not depend on $\varphi_{1}$. It is easily seen that the sets $\Psi(K) \cap[-2 \pi, 2 \pi)$ and $\Phi(\omega(\cdot, K))$ coincide.

Let $\varphi_{s} \notin \Phi(K), s=1, \ldots, p$, be such that $\varphi_{1} \in(-2 \pi, 0)$ and $\varphi_{1}<\cdots<\varphi_{p}<$ $\varphi_{1}+2 \pi=\varphi_{p+1}$. We set $a_{s}=z\left(\varphi_{s}, K\right), s=1, \ldots, p+1$. Consider the convex polygon $\Omega$ with the vertices $a_{1}, \ldots, a_{p}, a_{p+1}=a_{1}$, which is inscribed in the compact set $K$. Observe that some vertices with adjacent indices can coincide with each other. Let $e_{s}$ denote the outward unit normal to $\partial \Omega$ at the interior points (if they exist) of the segment $\left[a_{s}, a_{s+1}\right]$ (note that for some $\varphi(s) \in\left(\varphi_{s}, \varphi_{s+1}\right)$, we have $\left.e_{s}=e^{i \varphi(s)}\right)$. If $a_{s}=a_{s+1}$, then by $e_{s}$ we mean an arbitrary vector $e^{i \varphi}$, where $\varphi \in\left(\varphi_{s}, \varphi_{s+1}\right)$. The following statement has a simple geometric meaning.

Lemma 2.7. We have

$$
\sum_{s=1}^{p}\left|a_{s+1}-a_{s}\right| e_{s}=0
$$

Proof. Observe that

$$
\sum_{s=1}^{p}\left|a_{s+1}-a_{s}\right| e_{s}=\sum_{s=1}^{p}\left(a_{s+1}-a_{s}\right) e^{-i \pi / 2}=0 .
$$

Remark. Lemma 2.7 implies that for $\omega=\omega(\cdot, K)$, we have the identity

$$
\int_{0}^{2 \pi} e^{i \varphi} d \omega(\varphi)=0
$$


Conversely, suppose $\omega \in \Sigma$ satisfies this identity. Then (see [1, Section I.17, Theorem 24] or [2, Section I.2, Theorem 1.2.4]) the function

$$
H(\varphi)=A \cos \varphi+B \sin \varphi-\frac{1}{2 \pi} \int_{\varphi-2 \pi}^{\varphi}(\varphi-\theta) \sin (\varphi-\theta) d \omega(\theta)
$$

coincides with the support function $H(\varphi, K)$ of the compact convex set $K$ (for various $A, B \in \mathbb{R}$, the resulting compact sets are obtained from each other by shifting). We also have $\omega(\theta) \equiv \omega(\theta, K)$.

Let $\Sigma_{0}$ denote the subclass of the functions $\omega \in \Sigma$ that satisfy (2.17).

Lemma 2.8. Suppose $\Lambda=\left\{\lambda_{k}, n_{k}\right\}_{k=1}^{\infty}$ has an angular density and

$$
\int_{0}^{2 \pi} e^{i \varphi} d \omega_{\Lambda}(\varphi)=\mu
$$

Then for every $a>1$ and $r_{2}>r_{1}>0$ with $r_{2} / r_{1} \leq a$, we have

$$
\mathcal{N}\left(r_{2}, \Lambda\right)-\mathcal{N}\left(r_{1}, \Lambda\right)=\bar{\mu} \ln \left(\frac{r_{2}}{r_{1}}\right)+\varepsilon\left(r_{1}, r_{2}\right), \quad \varepsilon\left(r_{1}, r_{2}\right) \rightarrow 0, \quad r_{1} \rightarrow \infty .
$$

Proof. First, suppose $\omega_{\Lambda} \in \Sigma_{0}$. Then, as has been mentioned above, there exists a compact convex set $K$ satisfying $\omega_{\Lambda}(\varphi) \equiv \omega(\varphi, K)$.

We fix $\varepsilon>0$ and choose $\delta>0$ such that

$$
\left|e^{i \varphi}-e^{i \theta}\right| \leq \varepsilon /(4 s(K) \ln a), \quad \text { for all } \varphi, \theta \text { with }|\varphi-\theta|<\delta
$$

where $s(K)=\omega_{\Lambda}\left(\varphi_{1}+2 \pi\right)-\omega_{\Lambda}\left(\varphi_{1}\right)$ is the length of the boundary of $K$. Now we choose numbers $\varphi_{s} \notin \Phi\left(\omega_{\Lambda}\right), s=1, \ldots, p, \varphi_{1} \in(-2 \pi, 0), \varphi_{1}<\cdots<\varphi_{p}<\varphi_{1}+2 \pi=\varphi_{p+1}$, satisfying the following conditions:

$$
\text { 1) } \varphi_{s+1}-\varphi_{s}<\delta, s=1, \ldots, p, \quad \text { 2) } s(K)-P(\Omega)<\varepsilon /(4 \ln a),
$$

where $P(\Omega)$ is the perimeter of the convex polygon $\Omega$ with the vertices $a_{1}, \ldots, a_{p}, a_{p+1}=$ $a_{1}, a_{s}=z\left(\varphi_{s}, K\right), s=1, \ldots, p+1$.

Suppose that $\lambda_{k}=\left|\lambda_{k}\right| e^{i \psi_{k}}, \psi_{k} \in\left(\varphi_{1}, \varphi_{1}+2 \pi\right], k \geq 1$, and $\varphi(s) \in\left(\varphi_{s}, \varphi_{s+1}\right)$, $s=1, \ldots, p$, are such that the vector $e_{s}=e^{i \varphi(s)}$ is an outward normal vector to $\partial \Omega$ at the interior points of the segment $\left[a_{s}, a_{s+1}\right]$ (if there are no such points, then $\varphi(s) \in\left(\varphi_{s}, \varphi_{s+1}\right)$ is chosen arbitrarily). Then by condition 1) and the choice of $\delta>0$, we have

$$
\left|\sum_{\substack{\psi_{k} \in\left(\varphi_{s}, \varphi_{s+1}\right] \\ r_{1} \leq\left|\lambda_{k}\right|<r_{2}}}\left(\frac{n_{k}}{\lambda_{k}}-\frac{n_{k}}{\left|\lambda_{k}\right| e^{i \varphi(s)}}\right)\right| \leq \frac{\varepsilon}{4 s(K) \ln a} \sum_{\substack{\psi_{k} \in\left(\varphi_{s}, \varphi_{s+1}\right] \\ r_{1} \leq\left|\lambda_{k}\right|<r_{2}}} \frac{n_{k}}{\left|\lambda_{k}\right|} .
$$

Since $n\left(\Lambda\left(\varphi_{s}, \varphi_{s+1}\right]\right)=\omega_{\Lambda}\left(\varphi_{s+1}\right)-\omega_{\Lambda}\left(\varphi_{s}\right)$, this implies (by Lemma 2.6) that

$$
\left|\sum_{\substack{\psi_{k} \in\left(\varphi_{s}, \varphi_{s+1}\right] \\ r_{1} \leq\left|\lambda_{k}\right|<r_{2}}}\left(\frac{n_{k}}{\lambda_{k}}-\frac{n_{k}}{\left|\lambda_{k}\right| e^{i \varphi(s)}}\right)\right| \leq \frac{\varepsilon\left(\omega_{\Lambda}\left(\varphi_{s+1}\right)-\omega_{\Lambda}\left(\varphi_{s}\right)\right)}{4 s(K)}+\varepsilon_{s}\left(r_{1}, r_{2}\right),
$$

where $r_{2}>r_{1}>0, r_{2} / r_{1} \leq a$, and $\varepsilon_{s}\left(r_{1}, r_{2}\right) \rightarrow 0, r_{1} \rightarrow \infty$. Therefore,

$$
\begin{aligned}
& \sum_{s=1}^{p}\left|\sum_{\substack{\psi_{k} \in\left(\varphi_{s}, \varphi_{s+1}\right] \\
r_{1} \leq\left|\lambda_{k}\right|<r_{2}}}\left(\frac{n_{k}}{\lambda_{k}}-\frac{n_{k}}{\left|\lambda_{k}\right| e^{i \varphi(s)}}\right)\right| \leq \frac{\varepsilon}{4}+\widetilde{\varepsilon}\left(r_{1}, r_{2}\right) \leq \frac{\varepsilon}{2}, \\
& r_{2}>r_{1}>r_{0}(\varepsilon), \quad \frac{r_{2}}{r_{1}} \leq a .
\end{aligned}
$$


Let $\omega_{\Lambda}\left(\varphi_{s+1}\right)-\omega_{\Lambda}\left(\varphi_{s}\right)=\left|a_{s+1}-a_{s}\right|+\gamma_{s}$. Using Lemma 2.6 once again and applying Lemma 2.7, we have

$$
\begin{aligned}
\sum_{\substack{s=1 \\
\psi_{k} \in\left(\varphi_{s}, \varphi_{s+1}\right] \\
r_{1} \leq\left|\lambda_{k}\right|<r_{2}}}^{p} \frac{n_{k} \bar{e}_{s}}{\left|\lambda_{k}\right|} & =\ln \left(\frac{r_{2}}{r_{1}}\right) \sum_{s=1}^{p} \bar{e}_{s}\left(\left|a_{s+1}-a_{s}\right|+\gamma_{s}\right)+\hat{\varepsilon}\left(r_{1}, r_{2}\right) \\
& =\ln \left(\frac{r_{2}}{r_{1}}\right) \sum_{s=1}^{p} \bar{e}_{s} \gamma_{s}+\hat{\varepsilon}\left(r_{1}, r_{2}\right), \quad \hat{\varepsilon}\left(r_{1}, r_{2}\right) \rightarrow 0, \quad r_{1} \rightarrow \infty .
\end{aligned}
$$

By (2.18) and condition 2), this implies

$$
\begin{aligned}
& \mathcal{N}\left(r_{2}, \Lambda\right)-\mathcal{N}\left(r_{1}, \Lambda\right)=\varepsilon\left(r_{1}, r_{2}\right), \\
& \left|\varepsilon\left(r_{1}, r_{2}\right)\right| \leq \frac{\varepsilon}{2}+\ln \left(\frac{r_{2}}{r_{1}}\right)\left|\sum_{s=1}^{p} \bar{e}_{s} \gamma_{s}\right|+\left|\hat{\varepsilon}\left(r_{1}, r_{2}\right)\right| \leq \frac{3 \varepsilon}{4}+\ln a(s(K)-P(\Omega)) \leq \varepsilon,
\end{aligned}
$$

where $r_{2}>r_{1}>\widetilde{r}_{0}(\varepsilon), r_{2} / r_{1} \leq a$. This completes the proof of the lemma in the case where $\mu=0$.

Now, let $\mu=|\mu| e^{i \varphi(0)} \neq 0$. We set $\Lambda^{1}=\left\{-n e^{i \varphi(0)} /|\mu|, 1\right\}_{n=1}^{\infty}$ and $\Lambda^{2}=\Lambda^{1} \cup \Lambda$. The sequence $\Lambda^{1}$ lies on the half-line $\left\{\lambda=-t e^{i \varphi(0)}, t>0\right\}$ and has density $|\mu|$, and $\Lambda^{2}$ has the angular density $\omega_{\Lambda^{2}}=\omega_{\Lambda}+\omega_{\Lambda^{1}}$. It is easily seen that

$$
\int_{0}^{2 \pi} e^{i \varphi} d \omega_{\Lambda^{2}}(\varphi)=\int_{0}^{2 \pi} e^{i \varphi} d \omega_{\Lambda}(\varphi)+\int_{0}^{2 \pi} e^{i \varphi} d \omega_{\Lambda^{1}}(\varphi)=\mu-|\mu| e^{i \varphi(0)}=0 .
$$

By the above, we have

$$
\mathcal{N}\left(r_{2}, \Lambda^{2}\right)-\mathcal{N}\left(r_{1}, \Lambda^{2}\right)=\varepsilon^{\prime}\left(r_{1}, r_{2}\right), \quad \varepsilon^{\prime}\left(r_{1}, r_{2}\right) \rightarrow 0, \quad r_{1} \rightarrow \infty, \quad r_{2}>r_{1}>0, \quad r_{2} / r_{1} \leq a .
$$

Therefore, the definition of $\Lambda^{2}$ and Lemma 2.6 yield

$$
\mathcal{N}\left(r_{2}, \Lambda\right)-\mathcal{N}\left(r_{1}, \Lambda\right)=e^{-i \varphi(0)} \sum_{r_{1}|\mu| \leq n<r_{2}|\mu|} \frac{|\mu|}{n}+\varepsilon^{\prime}\left(r_{1}, r_{2}\right)=\bar{\mu} \ln \left(\frac{r_{2}}{r_{1}}\right)+\varepsilon\left(r_{1}, r_{2}\right),
$$

where $\varepsilon\left(r_{1}, r_{2}\right) \rightarrow 0, r_{1} \rightarrow \infty, r_{2}>r_{1}>0, r_{2} / r_{1} \leq a$. The lemma is proved.

Theorem 2.9. Let $\omega \in \Sigma_{0}$, and let $\Lambda^{1} \subseteq \Lambda^{2}$ be such that $n\left(r, \Lambda^{1}\right)<+\infty, r>0$, and for all admissible $\varphi, \psi \notin \Phi(\omega)$ we have the inequalities

$$
\underline{n}_{0}\left(\Lambda^{2}(\varphi, \psi)\right) \geq \omega(\psi)-\omega(\varphi), \quad \bar{n}_{0}\left(\Lambda^{1}(\varphi, \psi)\right) \leq \omega(\psi)-\omega(\varphi) .
$$

We also assume that for some $\varphi_{1}, \varphi_{2}, \varphi_{3} \in[-\pi, \pi)$ and $\psi_{j, p}>0, j=1,2,3, p \geq 1$, satisfying $\varphi_{1}<\varphi_{2}<\varphi_{3}, \varphi_{2}-\varphi_{1}<\pi, \varphi_{3}-\varphi_{2}<\pi, \varphi_{1}+2 \pi-\varphi_{3}<\pi, \psi_{j, p} \rightarrow 0, p \rightarrow \infty$, and $\varphi_{j}+\psi_{j, p} \notin \Phi(\omega), j=1,2,3, p \geq 1$, at least one of the following two conditions is fulfilled:

$$
\begin{aligned}
& \underline{n}_{0}\left(\Lambda^{2}\left(\varphi_{j}-\psi_{j, p}, \varphi_{j}+\psi_{j, p}\right)\right)>\omega\left(\varphi_{j}+\psi_{j, p}\right)-\omega\left(\varphi_{j}-\psi_{j, p}\right), \quad j=1,2,3, \quad p \geq 1 \\
& \bar{n}_{0}\left(\Lambda^{1}\left(\varphi_{j}-\psi_{j, p}, \varphi_{j}+\psi_{j, p}\right)\right)<\omega\left(\varphi_{j}+\psi_{j, p}\right)-\omega\left(\varphi_{j}-\psi_{j, p}\right), \quad j=1,2,3, \quad p \geq 1
\end{aligned}
$$

Then there exists a regularly distributed sequence $\Lambda$ with angular density $\omega$ such that $\Lambda^{1} \subseteq \Lambda \subseteq \Lambda^{2}$ and $\mathcal{N}(r, \Lambda) \rightarrow 0, r \rightarrow+\infty$.

Proof. By Theorem 2.4, there exists a sequence $\widetilde{\Lambda}$ with angular density $\omega$ such that $\Lambda^{1} \subseteq \tilde{\Lambda} \subseteq \Lambda^{2}$. First, suppose that (2.19) is true and $\Lambda^{3}=\Lambda^{2} \backslash \widetilde{\Lambda}$. Then

$$
\begin{aligned}
& \underline{n}_{0}\left(\Lambda^{3}\left(\varphi_{j}-\psi_{j, p}, \varphi_{j}+\psi_{j, p}\right)\right) \\
& \quad=\underline{n}_{0}\left(\Lambda^{2}\left(\varphi_{j}-\psi_{j, p}, \varphi_{j}+\psi_{j, p}\right)\right)-n\left(\widetilde{\Lambda}\left(\varphi_{j}-\psi_{j, p}, \varphi_{j}+\psi_{j, p}\right)\right) \\
& \quad>\omega\left(\varphi_{j}+\psi_{j, p}\right)-\omega\left(\varphi_{j}-\psi_{j, p}\right)-\left(\omega\left(\varphi_{j}+\psi_{j, p}\right)-\omega\left(\varphi_{j}-\psi_{j, p}\right)\right)=0 .
\end{aligned}
$$


Hence, Lemma 2.1 implies that $\Lambda^{3}$ is a sequence of general form. We set

$$
\gamma_{1}=-\mathcal{N}\left(2^{2}, \widetilde{\Lambda}\right), \quad \gamma_{m}=-\left(\mathcal{N}\left(2^{m+1}, \widetilde{\Lambda}\right)-\mathcal{N}\left(2^{m}, \widetilde{\Lambda}\right)\right), \quad m \geq 2 .
$$

Since $\omega \in \Sigma_{0}$, by Lemma 2.8 we have $\gamma_{m} \rightarrow 0, m \rightarrow \infty$. Then Lemma 2.5 shows that there exists a sequence $T \subset \Lambda^{3}$ of zero density with

$$
-\mathcal{N}\left(2^{l+1}, \Lambda\right)=-\mathcal{N}\left(2^{l+1}, \widetilde{\Lambda}\right)-\mathcal{N}\left(2^{l+1}, T\right)=\sum_{m=1}^{l} \gamma_{m}-\mathcal{N}\left(2^{l+1}, T\right) \rightarrow 0, \quad l \rightarrow \infty,
$$

where $\Lambda=\widetilde{\Lambda} \cup T$. The sequence $\Lambda$ (as well as $\widetilde{\Lambda}$ ) has an angular density $\omega \in \Sigma_{0}$. Furthermore, we have the embeddings $\Lambda^{1} \subseteq \Lambda \subseteq \Lambda^{2}$. Suppose that $r>0$ and that $l(r)$ is chosen so that $2^{l(r)} \leq r<2^{l(r)+1}$. Then, taking the above into account, by Lemma 2.8 we obtain

$$
\mathcal{N}(r, \Lambda)=\mathcal{N}\left(2^{l(r)}, \Lambda\right)+\left(\mathcal{N}(r, \Lambda)-N\left(2^{l(r)}, \Lambda\right)\right) \rightarrow 0, \quad r \rightarrow+\infty .
$$

In particular, this implies that $\Lambda$ is a regularly distributed sequence. This proves the theorem in the case under consideration. Now suppose that $(2.20)$ is fulfilled and $\Lambda^{4}=$ $\widetilde{\Lambda} \backslash \Lambda^{1}$. Then by Lemma 2.1 we have

$$
\begin{aligned}
& \quad \underline{n}_{0}\left(\Lambda^{4}\left(\varphi_{j}-\psi_{j, p}, \varphi_{j}+\psi_{j, p}\right)\right) \\
& \quad \geq n\left(\widetilde{\Lambda}\left(\varphi_{j}-\psi_{j, p}, \varphi_{j}+\psi_{j, p}\right)\right)-\bar{n}_{0}\left(\Lambda^{1}\left(\varphi_{j}-\psi_{j, p}, \varphi_{j}+\psi_{j, p}\right)\right)>0 .
\end{aligned}
$$

Thus, $\Lambda^{4}$ is a sequence of general form. We set

$$
\gamma_{1}=\mathcal{N}\left(2^{2}, \widetilde{\Lambda}\right), \quad \gamma_{m}=\mathcal{N}\left(2^{m+1}, \widetilde{\Lambda}\right)-\mathcal{N}\left(2^{m}, \widetilde{\Lambda}\right), \quad m \geq 2 .
$$

Then as above, we find a sequence $T \subset \Lambda^{4}$ of zero density such that for $\Lambda=\widetilde{\Lambda} \backslash T$ we have $\mathcal{N}(r, \Lambda) \rightarrow 0, r \rightarrow+\infty$. Furthermore, we have the embeddings $\Lambda^{1} \subseteq \Lambda \subseteq \Lambda^{2}$. The theorem is proved.

Consider some corollaries to Theorem 2.9. Let $\Lambda_{\mathbb{Z}}$ be a sequence consisting of all simple $\left(n_{k}=1\right)$ points $\lambda_{k}$ whose coordinates are integers. We have the following statement (compare with [1, Section II.1, Theorem 3] and [2, Section I.3.1]).

Corollary 1. Let $\omega \in \Sigma_{0}$. There exists a regularly distributed set $\Lambda \subset \Lambda_{\mathbb{Z}}$ with angular density $\omega$ such that $\mathcal{N}(r, \Lambda) \rightarrow 0, r \rightarrow+\infty$.

Proof. It is easily seen that for every admissible $\varphi, \psi$, we have $\underline{n}_{0}\left(\Lambda_{\mathbb{Z}}(\varphi, \psi)\right)=+\infty$. It remains to apply Theorem 2.9 (with $\Lambda^{1}=\varnothing$ and $\Lambda^{2}=\Lambda_{\mathbb{Z}}$ ). The corollary is proved.

We say that $\omega \in \Sigma_{0}$ is a function of general form if on the interval $[0,2 \pi]$ it takes more than three pairwise distinct values. Let $K$ be a compact convex set for which we have the identity $\omega(\varphi) \equiv \omega(\varphi, K)$. If $K$ is a point, then $\omega(\varphi) \equiv 0$. If $K$ is a line segment, then $\omega$ takes exactly three pairwise distinct values on $[0,2 \pi]$. In other cases (i.e., where $K$ is the closure of a domain), $\omega$ takes more than three pairwise distinct values on the interval $[0,2 \pi]$. If $\Lambda$ has an angular density $\omega \in \Sigma_{0}$, then it is easily seen that $\Lambda$ is a sequence of general form if and only if $\omega=\omega_{\Lambda}$ is a function of general form.

Thus, Theorem 2.9 implies the next claim.

Corollary 2. Suppose $\omega \in \Sigma_{0}$ is a function of general form and $\Lambda^{2}$ is such that $\underline{n}_{0}\left(\Lambda^{2}(\varphi, \psi)\right) \geq \omega(\psi)-\omega(\varphi)$ for all admissible $\varphi, \psi \notin \Phi(\omega)$. Then there exists a regularly distributed set $\Lambda \subseteq \Lambda^{2}$ with angular density $\omega$ such that $\mathcal{N}(r, \Lambda) \rightarrow 0, r \rightarrow+\infty$.

The following statement contains the main result of [5] as a special case (for $\rho(r) \equiv 1$ ). 
Corollary 3. Suppose that $\omega \in \Sigma_{0}$ and $\Lambda^{1}=\left\{\lambda_{k}, n_{k}\right\}_{k=1}^{\infty}$ are such that $n\left(r, \Lambda^{1}\right)<+\infty$, $r>0$, and for all admissible $\varphi, \psi \notin \Phi(\omega)$ we have $\bar{n}_{0}\left(\Lambda^{1}(\varphi, \psi)\right) \leq \omega(\psi)-\omega(\varphi)$. Then there exists a regularly distributed set $\Lambda$ with angular density $\omega$ such that $\Lambda^{1} \subseteq \Lambda \subseteq$ $\Lambda^{1} \cup \Lambda_{\mathbb{Z}}$ and $\mathcal{N}(r, \Lambda) \rightarrow 0, r \rightarrow+\infty$.

Here is an example showing that conditions (2.19) and (2.20) in Theorem 2.9 cannot be lifted. Let $\Lambda^{2}=\Lambda^{-} \cup \Lambda^{+}$, where $\Lambda^{-}=\{-p\}_{p=1}^{+\infty}, \Lambda^{+}=\left\{\lambda_{k}\right\}_{k=2}^{+\infty}$, and

$$
\lambda_{k}=k \ln k \frac{\ln k+i(-1)^{\sigma(k)}}{1+\ln ^{2} k}, \quad \sigma(k)=0,1 .
$$

The sequence $\Lambda^{2}$ has the angular density $\omega=\omega(\cdot, K) / 2 \pi \in \Sigma_{0}$, where $K$ is the segment $[-i \pi, i \pi]$ of the imaginary axis. These $\omega$ and $\Lambda^{2}$ satisfy all the conditions of Corollary 2 except one: the function $\omega$ is not a function of general form. We show that the assertion of Corollary 2 fails in this case. We have

$$
\frac{1}{\lambda_{k}}=\frac{\ln k-i(-1)^{\sigma(k)}}{k \ln k}=\frac{1}{k}-i \frac{(-1)^{\sigma(k)}}{k \ln k} .
$$

By (2.15), we have

$$
\operatorname{Re} \mathcal{N}\left(\left|\lambda_{k}\right|, \Lambda^{2}\right)=-\sum_{k \leq p<\left|\lambda_{k}\right|} \frac{1}{p} \geq-\ln \frac{\left|\lambda_{k}\right|}{k}-\widetilde{\beta}(k) \rightarrow 0, \quad k \rightarrow+\infty .
$$

This implies that $\operatorname{Re} \mathcal{N}(r, \Lambda) \rightarrow 0, r \rightarrow+\infty$. We choose numbers $\sigma(k)$ so that

$$
\limsup _{r \rightarrow+\infty}|\mathcal{N}(r, \Lambda)|=\limsup _{r \rightarrow+\infty}|\operatorname{Im} \mathcal{N}(r, \Lambda)|<+\infty .
$$

First, we note the following:

$$
\begin{aligned}
\frac{\ln 2+\beta_{0}(m)}{(m+1)} & =\sum_{2^{m} \leq k<2^{m+1}} \frac{1}{k(m+1)} \\
& \leq \sum_{2^{m} \leq k<2^{m+1}} \frac{1}{k \ln k} \leq \sum_{2^{m} \leq k<2^{m+1}} \frac{1}{k m}=\frac{\ln 2+\beta_{0}(m)}{m},
\end{aligned}
$$

where $\beta_{0}(m) \rightarrow 0, m \rightarrow \infty$. Thus, there exist numbers $m(j), j \geq 1$, such that for $\sigma(k)=0,1<k<2^{m(1)}, 2^{m(2 l-1)} \leq k<2^{m(2 l)}$ and $\sigma(k)=1,2^{m(2 l)} \leq k<2^{m(2 l+1)}$, $l \geq 1$, we have the relations

$$
3 \leq \sum_{2^{m(1)} \leq k<2^{m(2 l)}} \frac{(-1)^{\sigma(k)}}{k \ln k} \leq 4, \quad 0 \leq \sum_{2^{m(1)} \leq k<2^{m(2 l+1)}} \frac{(-1)^{\sigma(k)}}{k \ln k} \leq 1, \quad l \geq 1 .
$$

Hence, we get (2.21) and the inequality

$$
\sum_{2^{m(2 l-1)} \leq k<2^{m(2 l)}} \frac{(-1)^{\sigma(k)}}{k \ln k}=\sum_{2^{m(2 l-1)} \leq k<2^{m(2 l)}} \frac{1}{k \ln k} \geq 2 .
$$

Let $T \subseteq \Lambda^{2}$ be a sequence with zero density and let $\Lambda=\Lambda^{2} \backslash T$. The identity $n\left(\Lambda^{+}\right)=1$ and Lemma 2.1 imply that for any $\varepsilon>0$ there exists a number $m_{0}(\varepsilon)$ such that

$$
n\left(r_{m+1}, T\right)-n\left(r_{m}, T\right) \leq \varepsilon 2^{m}, \quad m \geq m_{0}(\varepsilon), \quad r_{l}=\left|\lambda_{2^{l}}\right|, \quad l \geq 1 .
$$

Therefore, by (2.15) we have

$$
\left|\operatorname{Im}\left(\mathcal{N}\left(r_{m+1}, T\right)-\mathcal{N}\left(r_{m}, T\right)\right)\right| \leq \frac{\ln (1+\varepsilon)+\beta_{1}(m)}{m}, m \geq m_{0}(\varepsilon), \quad \beta_{1}(m) \rightarrow 0, m \rightarrow \infty .
$$


Thus, taking (2.22) into account, for sufficiently small $\varepsilon>0$ we have

$$
\begin{array}{r}
\operatorname{Im}\left(\mathcal{N}\left(r_{m+1}, \Lambda\right)-\mathcal{N}\left(r_{m}, \Lambda\right)\right) \geq \frac{\ln 2+\beta_{0}(m)}{(m+1)}-\frac{\ln (1+\varepsilon)+\beta_{1}(m)}{m} \geq \frac{\ln 2+\beta_{0}(m)}{2 m}, \\
m \geq m_{1}(\varepsilon),
\end{array}
$$

where $m(2 l-1) \leq m<m(2 l)$. Using this and (2.22), (2.23), we obtain

$$
\operatorname{Im}\left(\mathcal{N}\left(r_{m(2 l-1)}, \Lambda\right)-\mathcal{N}\left(r_{m(2 l)}, \Lambda\right)\right) \geq 1 .
$$

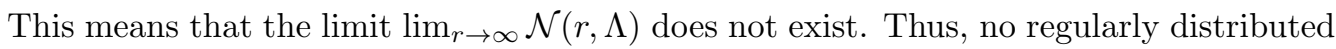
set $\Lambda \subseteq \Lambda^{2}$ with angular density $\omega$ can exist.

In the above example, it was important that $\sum_{k \geq 1}\left|\operatorname{Im}\left(1 / \lambda_{k}\right)\right|=+\infty$. In this connection, we have the following proposition.

Lemma 2.10. Suppose that $\Lambda=\left\{\lambda_{k}, n_{k}\right\}_{k=1}^{\infty}$ has an angular density $\omega \in \Sigma_{0}$ and $\sum_{k \geq 1}\left|\operatorname{Im}\left(n_{k} / \lambda_{k}\right)\right|<+\infty$. Then $\omega=\omega(\cdot, K) / 2 \pi$, where $K$ is the segment $[-i \pi, i \pi]$ of the imaginary axis $(K=\{0\}$ if $\tau=0)$, and there is a set $T \subseteq \Lambda$ of zero density for which the limit $\lim _{r \rightarrow \infty}(\mathcal{N}(r, \Lambda)-\mathcal{N}(r, T))$ exists and $\operatorname{Re}(\mathcal{N}(r, \Lambda)-\mathcal{N}(r, T)) \rightarrow 0$, $r \rightarrow \infty$.

Proof. Let $\varphi \in(0, \pi / 2)$ and $\lambda_{k} \in \Gamma[\varphi, \pi-\varphi](\Gamma[-\pi+\varphi,-\varphi])$. Then

$$
\frac{1}{\left|\lambda_{k}\right|} \leq\left|\operatorname{Re} \frac{1}{\lambda_{k}}\right|+\left|\operatorname{Im} \frac{1}{\lambda_{k}}\right| \leq c\left|\operatorname{Im} \frac{1}{\lambda_{k}}\right| .
$$

Hence, the assumption implies $n(\Lambda[\varphi, \pi-\varphi])=n(\Lambda[-\pi+\varphi,-\varphi])=0$. Therefore, for some $\tau_{1}, \tau_{2} \geq 0$ and every $\varphi \in(0, \pi / 2)$ we have $n(\Lambda(-\varphi, \varphi))=\tau_{1}$ and $n(\Lambda(\pi-\varphi, \pi+\varphi))=$ $\tau_{2}$. Since $\omega \in \Sigma_{0}$, it follows that $\tau_{1} e^{i 0}+\tau_{2} e^{i \pi}=0$, i.e., $\tau_{1}=\tau_{2}=\tau$. Thus, we have $\omega=\omega(\cdot, K) / 2 \pi$, where $K$ is the segment $[-i \tau \pi, i \tau \pi]$ of the imaginary axis.

We fix $\varphi \in(0, \pi / 2)$. Let $\Lambda_{\varphi}^{+}=\left\{\xi_{j}\right\}_{j \geq 1}$ be a positive sequence consisting of the inverse values of the numbers $\operatorname{Re}\left(1 / \lambda_{k}\right)$, where $\lambda_{k} \in \Gamma(-\varphi, \varphi)$. Also we assume that the number of occurrences of each $\xi_{j}$ in $\Lambda_{\varphi}^{+}$coincides with the total multiplicity of the points $\lambda_{k}$ for which $\left(\operatorname{Re}\left(1 / \lambda_{k}\right)\right)^{-1}=\xi_{j}$. The sequence $\Lambda_{\varphi}^{-}$is defined similarly: it lies in the left half-plane and is constructed by $\Lambda(\pi-\varphi, \pi+\varphi)$.

If $\xi_{j}=\left(\operatorname{Re}\left(1 / \lambda_{k}\right)\right)^{-1}, \lambda_{k} \in \Gamma(-\varphi, \varphi)$, then $\left|\lambda_{k}\right| \leq \xi_{j} \leq\left|\lambda_{k}\right| / \cos \varphi$. Therefore, we have $n(r \cos \varphi, \Lambda(-\varphi, \varphi)) \leq n\left(r, \Lambda_{\varphi}^{+}\right) \leq n(r, \Lambda(-\varphi, \varphi))$. Then $\bar{n}\left(\Lambda_{\varphi}^{+}\right) \leq n(\Lambda(-\varphi, \varphi))=\tau$ and $\underline{n}\left(\Lambda_{\varphi}^{+}\right) \geq n(\Lambda(-\varphi, \varphi)) \cos \varphi=\tau \cos \varphi$. For every $0<\tilde{\varphi}<\varphi<\pi / 2$, we have the embed$\operatorname{ding} \Lambda_{\tilde{\varphi}}^{+} \subseteq \Lambda_{\varphi}^{+}$. Thus, $\underline{n}\left(\Lambda_{\varphi}^{+}\right) \geq \underline{n}\left(\Lambda_{\tilde{\varphi}}^{+}\right) \geq \tau \cos \tilde{\varphi}$. Hence, the previous considerations imply that $\underline{n}\left(\Lambda_{\varphi}^{+}\right)=\bar{n}\left(\Lambda_{\tilde{\varphi}}^{+}\right)=\tau$, i.e., the sequence $\Lambda_{\varphi}^{+}$has density $\tau$. A similar statement is true for $\Lambda_{\varphi}^{-}$.

Now we can apply [7, Lemma 4]. In accordance with it, there exists a set $\widetilde{T} \subseteq \Lambda_{\varphi}^{+} \cup \Lambda_{\varphi}^{-}$ of zero density such that

$$
\lim _{r \rightarrow \infty}\left(\mathcal{N}\left(r, \Lambda_{\varphi}^{+} \cup \Lambda_{\varphi}^{-}\right)-\mathcal{N}(r, \widetilde{T})\right)=0 .
$$

Let $T=T^{\prime} \cup \Lambda[\varphi, \pi-\varphi] \cup \Lambda[-\pi+\varphi,-\varphi]$, where $T^{\prime}$ is the sequence in the union $\Lambda(\varphi, \pi-$ $\varphi) \cup \Lambda(\pi-\varphi, \pi+\varphi)$ that corresponds to $\widetilde{T}$. Then $T$ has zero density. By construction, we have $\lim _{r \rightarrow \infty}(\operatorname{Re}(\mathcal{N}(r, \Lambda)-\mathcal{N}(r, T)))=0$. Since $\sum_{k \geq 1}\left|\operatorname{Im}\left(n_{k} / \lambda_{k}\right)\right|<+\infty$, this implies that the limit $\lim _{r \rightarrow \infty}(\mathcal{N}(r, \Lambda)-\mathcal{N}(r, T))$ exists. The lemma is proved.

At the end of the section we present another example containing a method for constructing a regular set (see [1, Section II.1]) by a given sequence of positive numbers. Suppose $\omega \in \Sigma_{0}$ and $\mu_{p}>0, \mu_{p+1}-\mu_{p} \geq h>0, p \geq 1, \widetilde{\Lambda}=\left\{\mu_{p}, 1\right\}_{p=1}^{\infty}$. We assume 
that $\tau=\underline{n}(r, \tilde{\Lambda}) \geq \omega(2 \pi)-\omega(0)$ if $\omega$ is a function of general form, and $\tau>\omega(2 \pi)-\omega(0)$ otherwise.

Let $\varphi_{s}^{j}, s=1, \ldots, s(j)+1, j \geq 1$ be the same as in the proof of Theorem 2.4. We fix $j \geq 1$ and put $\widetilde{\Lambda}_{0}^{j}=\varnothing$. For each $s=1, \ldots, s(j)$, we use Lemma 2.2 to find a sequence $\widetilde{\Lambda}_{s}^{j} \subseteq \widetilde{\Lambda} \backslash\left(\bigcup_{l=0}^{s-1} \widetilde{\Lambda}_{l}^{j}\right), \widetilde{\Lambda}_{s}^{j}=\left\{\mu_{p(s, m)}, 1\right\}_{m=1}^{\infty}$, such that

$$
\lim _{r \rightarrow \infty} n\left(r, \widetilde{\Lambda}_{s}^{j}\right) / r=\omega\left(\varphi_{s+1}^{j}\right)-\omega\left(\varphi_{s}^{j}\right) .
$$

This can be done because

$$
\underline{n}\left(\tilde{\Lambda} \backslash\left(\bigcup_{l=0}^{s-1} \tilde{\Lambda}_{l}^{j}\right)\right) \geq \omega\left(\varphi_{1}^{j}+2 \pi\right)-\omega\left(\varphi_{1}^{j}\right)-\sum_{l=1}^{s-1} n\left(\widetilde{\Lambda}_{l}^{j}\right)=\sum_{l=s}^{s(j)}\left(\omega\left(\varphi_{l+1}^{j}\right)-\omega\left(\varphi_{l}^{j}\right)\right) .
$$

We set $\Lambda_{s}^{j}=\left\{\mu_{p(s, m)} e^{i \varphi_{s+1}^{j}}, 1\right\}_{m=1}^{\infty}$. As in Theorem 2.4, we "glue" a sequence $\Lambda$ with angular density $\omega$ from the sequences $\Lambda_{s}^{j}$. If $\omega$ is a function of general form, then $\Lambda$ is a sequence of general form. Then, as in Theorem 2.9, we find a set $T \subseteq \Lambda$ of zero density such that $\mathcal{N}(r, \Lambda \backslash T) \rightarrow 0, r \rightarrow+\infty$. Otherwise, we have

$$
\underline{n}\left(\tilde{\Lambda} \backslash\left(\bigcup_{l=1}^{s(j)} \tilde{\Lambda}_{l}^{j}\right)\right)=\tau-(\omega(2 \pi)-\omega(0))=\tilde{\tau}>0 .
$$

Then, using Lemma 2.2, we build, with the help of $\widetilde{\Lambda} \backslash\left(\bigcup_{l=1}^{s(j)} \widetilde{\Lambda}_{l}^{j}\right)$, a sequence $\Lambda^{\prime}$ of general form that lies on three half-lines and has the density $\widetilde{\tau} / 3$ on the each of them. Next, we find, as in Theorem 2.9, a set $T \subseteq \Lambda^{\prime}$ of zero density such that $\mathcal{N}(r, \Lambda \cup T) \rightarrow 0, r \rightarrow+\infty$. In both cases, we get a regularly distributed set $\left\{\lambda_{k}, 1\right\}_{k=1}^{\infty}$ with angular density $\omega$; by the condition on $\mu_{p}$, this is a regular set. We also have $\left\{\left|\lambda_{k}\right|, 1\right\}_{k=1}^{\infty} \subseteq \widetilde{\Lambda}$.

\section{$\S 3$. Splitting And the ASymptotic Behavior OF ENTIRE FUNCTIONS WITH A MEASURABLE SEQUENCE OF ZEROS}

Regularly distributed sets are closely related to functions of regular growth. Let $f$ be an entire function of exponential type, i.e., there exist $A>0$ and $B>0$ such that

$$
\ln |f(\lambda)| \leq A+B|\lambda|, \quad \lambda \in \mathbb{C} .
$$

The function

$$
h_{f}(\lambda)=\limsup _{t \rightarrow \infty} \ln |f(t \lambda)| / t, \quad \lambda \in \mathbb{C},
$$

is called the upper indicator of $f$ (or simply the indicator). The indicator $h_{f}$ is a positive homogeneous convex function of order one that coincides with the support function of some compact convex set $K$, which is called the indicator diagram of $f$ (see, e.g., [13, Section I.5, Theorem 5.4]). The compact set that is complex conjugate to $K$ is called the conjugate diagram.

We say (see [1, Chapter III]) that $f$ is of regular growth if

$$
h_{f}(\lambda)=\lim _{t \rightarrow \infty, t \notin E} \ln |f(t \lambda)| / t, \quad \lambda \in \mathbb{C},
$$

where $E$ is a set of relative measure zero on the half-line $(0,+\infty)$, i.e., the Lebesgue measure of its intersection with the interval $(0, r)$ is infinitely small in comparison with $r$ as $r \rightarrow+\infty$. Such a set is called an $E^{0}$-set. The regularity of growth of $f$ is equivalent to the asymptotic identity

$$
\ln |f(\lambda)|=h_{f}(\lambda)+\alpha(\lambda), \quad \lambda \in \mathbb{C}, \quad \lim _{|\lambda| \rightarrow \infty, \lambda \notin \mathcal{I}_{f}} \alpha(\lambda) /|\lambda|=0,
$$


where $\mathcal{I}_{f}$ is some $C^{0}$-set. We recall (see [1, Section II.1]) that $\mathcal{R} \subset \mathbb{C}$ is called a $C^{0}$-set if it can be covered by disks $B\left(z_{j}, r_{j}\right), j \geq 1$, such that

$$
\lim _{r \rightarrow \infty} \frac{1}{r} \sum_{\left|z_{j}\right|<r} r_{j}=0
$$

Let $\Lambda=\left\{\lambda_{k}, n_{k}\right\}_{k=1}^{\infty}$, and let $f(\lambda, \Lambda)$ be the canonical product

$$
f(\lambda, \Lambda)=\prod_{k=1}^{\infty}\left(1-\frac{\lambda}{\lambda_{k}}\right)^{n_{k}} \exp \frac{n_{k} \lambda}{\lambda_{k}} .
$$

The function $f(\lambda, \Lambda)$ is of regular growth if and only if (see [1, III.3, Theorem 4 and II.1, Theorem 2]) $\Lambda$ is a regularly distributed set. In addition, its angular density $\omega$ lies in $\Sigma_{0}$ and we have $\left(\lambda=r e^{i \varphi}\right)$

$$
h_{f}(\lambda)=r H(\varphi)=\operatorname{Re}(\lambda \nu(\Lambda))-r \int_{\varphi-2 \pi}^{\varphi}(\varphi-\theta) \sin (\varphi-\theta) d \omega(\theta),
$$

where $\nu(\Lambda)=\lim _{r \rightarrow \infty} \mathcal{N}(r, \Lambda)$. If $K$ is the indicator diagram of the function $f(\lambda, \Lambda)$, then (see [1, II.1, (2.07)]) we have $\omega(\theta) \equiv \omega(\theta, K) / 2 \pi$ and $H(\varphi) \equiv H(\varphi, K)$.

We also set

$$
F(\lambda, \Lambda)=\prod_{\left|\lambda_{k}\right|<|\lambda|} \exp \frac{n_{k} \lambda}{\lambda_{k}}=\exp (\mathcal{N}(|\lambda|, \Lambda) \lambda) .
$$

Let $m \geq 1$ and $\tau_{m}=n\left(2^{m}, \Lambda\right) / 2^{m}$. By Cartan's theorem (see [1, Section I.7, Theorem 10]), outside a union of disks with the total sum of radii $2 e \tau_{m+1} 2^{m+1}$ we have the estimate

$$
\prod_{\left|\lambda_{k}\right|<2^{m+1}}\left|\lambda-\lambda_{k}\right|^{n_{k}} \geq\left(\tau_{m+1} 2^{m+1}\right)^{n\left(2^{m+1}, \Lambda\right)} .
$$

Let $B\left(z_{m, j}, r_{m, j}\right), j=1, \ldots, j(m)$, be all the exceptional disks that intersect the annulus $B\left(0,2^{m}\right) \backslash B\left(0,2^{m-1}\right)$. Then $\sum_{j=1}^{j(m)} r_{m, j} 2 e \tau_{m+1} 2^{m+1}$. We set

$$
\mathcal{I}(\Lambda)=\bigcup_{m=1, j=1}^{\infty, j(m)} B\left(z_{m, j}, r_{m, j}\right) .
$$

Lemma 3.1. Suppose $\Lambda=\left\{\lambda_{k}, n_{k}\right\}_{k=1}^{\infty}$ has zero density. Then $\mathcal{I}(\Lambda)$ is a $C^{0}$-set.

Proof. By assumption, there exists $m_{0}$ such that $\tau_{m} \leq 1 / 32, m \geq m_{0}$. Suppose $r \geq 2^{m_{0}}$ and $m \geq m_{0}$ satisfy the inequalities $2^{m} \leq r<2^{m+1}$. We assume that $\left|z_{l, j}\right|<r$ and $l>m+1$. Then by the definition of the disks $B\left(z_{l, j}, r_{l, j}\right)$ we have the estimates

$$
2^{l-1}-2^{m+1} \leq 2^{l-1}-r \leq r_{l, j} \leq 2 e \tau_{l+1} 2^{l+1} \leq \tau_{l+1} 2^{l+3} \leq 2^{l-2} .
$$

This implies that $l \leq m+3$. Thus, we have

$$
\frac{1}{r} \sum_{\left|z_{l, j}\right|<r} r_{l, j} \leq \frac{1}{2^{m}} \sum_{l=1, j=1}^{m+2, j(m)} r_{l, j} \leq \frac{1}{2^{m}} \sum_{l=1}^{m+3} 2 e \tau_{l+1} 2^{l+1} \leq \frac{1}{2^{m-4}} \sum_{l=1}^{m+3} \tau_{l+1} 2^{l} .
$$

We fix $\varepsilon>0$. In accordance with the assumption, we can find $m_{1} \geq m_{0}$ such that $\tau_{m} \leq \varepsilon / 2^{8}, m \geq m_{1}$. Then

$$
\frac{1}{r} \sum_{\left|z_{l, j}\right|<r} r_{l, j} \leq \frac{1}{2^{m-4}} \sum_{l=1}^{m_{1}} \tau_{l+1} 2^{l}+\frac{\varepsilon}{2^{m-4}} \sum_{l=m_{1}+1}^{m+3} 2^{l-8}, \quad r \geq 2^{m_{1}} .
$$


Hence, we get

$$
\limsup _{r \rightarrow \infty} \frac{1}{r} \sum_{\left|z_{j}\right|<r} r_{j} \leq \varepsilon .
$$

Since $\varepsilon>0$ is arbitrary, the lemma is proved.

Lemma 3.2. Suppose $\Lambda=\left\{\lambda_{k}, n_{k}\right\}_{k=1}^{\infty}$ has zero density. Then

$$
\begin{aligned}
\ln |f(\lambda, \Lambda)| & =\ln |F(\lambda, \Lambda)|+\alpha(\lambda), \quad \lambda \in \mathbb{C}, \\
\lim _{\substack{|\lambda| \rightarrow \infty \\
\lambda \notin \mathcal{I}(\Lambda)}} \alpha(\lambda) /|\lambda| & =0, \quad \limsup _{|\lambda| \rightarrow \infty} \alpha(\lambda) /|\lambda|=0 .
\end{aligned}
$$

Proof. Suppose $m \geq 1,2^{m-1} \leq|\lambda|<2^{m}$, and

$$
\begin{aligned}
& f_{1, m}(\lambda, \Lambda)=\prod_{\left|\lambda_{k}\right|<2^{m+1}}\left(1-\frac{\lambda}{\lambda_{k}}\right)^{n_{k}}, \quad f_{2, m}(\lambda, \Lambda)=\prod_{|\lambda| \leq\left|\lambda_{k}\right|<2^{m+1}} \exp \frac{n_{k} \lambda}{\lambda_{k}}, \\
& f_{3, m}(\lambda, \Lambda)=\prod_{\left|\lambda_{k}\right| \geq 2^{m+1}}\left(1-\frac{\lambda}{\lambda_{k}}\right)^{n_{k}} \exp \frac{n_{k} \lambda}{\lambda_{k}} .
\end{aligned}
$$

We have

$$
\ln |f(\lambda, \Lambda)|=\ln |F(\lambda, \Lambda)|+\ln \left|f_{1, m}(\lambda, \Lambda)\right|+\ln \left|f_{2, m}(\lambda, \Lambda)\right|+\ln \left|f_{3, m}(\lambda, \Lambda)\right| .
$$

Using (3.1), we get

$$
\begin{array}{r}
|F(\lambda, \Lambda)| \geq\left(\tau_{m+1} 2^{m+1}\right)^{n\left(2^{m+1}, \Lambda\right)} \prod_{\left|\lambda_{k}\right|<2^{m+1}}\left|\lambda_{k}\right|^{-n_{k}} \geq\left(\tau_{m+1}\right)^{n\left(2^{m+1}, \Lambda\right)}, \\
\lambda \notin \mathcal{I}(\Lambda) .
\end{array}
$$

This implies

$$
\ln \left|f_{1, m}(\lambda, \Lambda)\right| \geq \tau_{m+1} 2^{m+1} \ln \tau_{m+1}, \quad \lambda \notin \mathcal{I}(\Lambda) .
$$

Moreover, recalling that $\lambda_{k} \neq 0$ (i.e., $n(r, \Lambda)=0$ for small $r>0$ ), we have

$$
\begin{aligned}
\ln \left|f_{1, m}(\lambda, \Lambda)\right| & \leq \sum_{\left|\lambda_{k}\right|<2^{m+1}} n_{k} \ln \left(1+\frac{|\lambda|}{\left|\lambda_{k}\right|}\right)=\int_{0}^{2^{m+1}} \ln \left(1+\frac{|\lambda|}{r}\right) d n(r, \Lambda) \\
& =n\left(2^{m+1}, \Lambda\right) \ln \left(1+\frac{|\lambda|}{2^{m+1}}\right)+|\lambda| \int_{0}^{2^{m+1}} \frac{n(r, \Lambda) d r}{r(r+|\lambda|)} \\
& \leq n\left(2^{m+1}, \Lambda\right)+\int_{0}^{2^{m+1}} \frac{n(r, \Lambda) d r}{r} .
\end{aligned}
$$

Since the sequence $\left\{\left|\lambda_{k}\right|, n_{k}\right\}_{k=1}^{\infty}$ has zero density, Lemma 2.6 yields

$$
\sum_{|\lambda| \leq\left|\lambda_{k}\right|<2^{m+1}} \frac{n_{k}}{\left|\lambda_{k}\right|} \leq \sum_{2^{m-1} \leq\left|\lambda_{k}\right|<2^{m+1}} \frac{n_{k}}{\left|\lambda_{k}\right|}=\beta_{m} \rightarrow 0, \quad m \rightarrow \infty .
$$

Therefore,

$$
|\ln | f_{2, m}(\lambda, \Lambda)|| \leq \beta_{m}|\lambda|, \quad \lambda \in \mathbb{C}, \quad \beta_{m} \rightarrow 0, m \rightarrow \infty .
$$

Finally, we consider the function $f_{3, m}$. If $\left|\lambda_{k}\right| \geq 2^{m+1}$, then $|\lambda| /\left|\lambda_{k}\right|<1 / 2$. Hence,

$$
|\ln |\left(1-\frac{\lambda}{\lambda_{k}}\right) \exp \frac{\lambda}{\lambda_{k}}|| \leq\left|\ln \left(\left(1-\frac{\lambda}{\lambda_{k}}\right) \exp \frac{\lambda}{\lambda_{k}}\right)\right| \leq \sum_{p=2}^{\infty} \frac{|\lambda|^{p}}{p\left|\lambda_{k}\right|^{p}}
$$


(we mean the principal branch of the logarithm). This implies

$$
|\ln | f_{3, m}(\lambda, \Lambda)|| \leq\left|\ln f_{3, m}(\lambda, \Lambda)\right| \leq \sum_{\left|\lambda_{k}\right| \geq 2^{m+1}} \frac{n_{k}|\lambda|^{2}}{\left|\lambda_{k}\right|^{2}} .
$$

Thus, since $n(r, \Lambda) / r \rightarrow 0, r \rightarrow \infty$, we have

$$
|\ln | f_{3, m}(\lambda, \Lambda)|| \leq|\lambda|^{2} \int_{2^{m+1}}^{\infty} \frac{d n(r, \Lambda)}{r^{2}} \leq-\frac{n\left(2^{m+1}, \Lambda\right)|\lambda|^{2}}{2^{2 m+2}}+2|\lambda|^{2} \int_{2^{m+1}}^{\infty} \frac{n(r, \Lambda)}{r^{3}} d r
$$

Let $\varepsilon>0$. We choose a number $m(\varepsilon)$ satisfying the following conditions: $n(r, \Lambda) / r \leq$ $\varepsilon / 4$ for $r \geq 2^{m(\varepsilon)}, \tau_{m} \ln \tau_{m} \geq-\varepsilon / 4, m \geq m(\varepsilon)$, and $\beta_{m} \leq \varepsilon, m \geq m(\varepsilon)$. Then

$$
\begin{aligned}
\int_{0}^{2^{m+1}} \frac{n(r, \Lambda) d r}{r} & =\int_{0}^{2^{m(\varepsilon)}} \frac{n(r, \Lambda) d r}{r}+\int_{2^{m(\varepsilon)}}^{2^{m+1}} \frac{n(r, \Lambda) d r}{r} \leq A(\varepsilon)+\frac{\varepsilon 2^{m+1}}{4}, \\
\int_{2^{m+1}}^{\infty} \frac{n(r, \Lambda) d r}{r^{3}} & \leq \frac{\varepsilon}{4} \int_{2^{m+1}}^{\infty} \frac{d r}{r^{2}}=\frac{\varepsilon}{2^{m+3}} .
\end{aligned}
$$

Hence, taking (3.3)-(3.5) into account, for all $\lambda \in \mathbb{C}$ with $|\lambda| \geq 2^{m(\varepsilon)}$ we obtain

$$
\alpha(\lambda) \leq \frac{\varepsilon}{2} 2^{m+1}+A(\varepsilon)+\varepsilon|\lambda|+|\lambda|^{2} \frac{\varepsilon}{2^{m+2}},
$$

where $2^{m-1} \leq|\lambda|<2^{m}$. Therefore,

$$
\alpha(\lambda) \leq 2 \varepsilon|\lambda|+\varepsilon|\lambda|+\varepsilon|\lambda|=4 \varepsilon|\lambda| .
$$

Now, applying (3.2), (3.4), and (3.5), we get

$$
\alpha(\lambda) \geq-2^{m+1} \frac{\varepsilon}{4}-\varepsilon|\lambda|-|\lambda|^{2} \frac{\varepsilon}{2^{m+2}} \geq-\varepsilon|\lambda|-\varepsilon|\lambda|-\varepsilon|\lambda|=-3 \varepsilon|\lambda|,
$$

where $\lambda \notin \mathcal{I}(\Lambda), 2^{m-1} \leq|\lambda|<2^{m}$, and $m \geq m(\varepsilon)$. Since $\varepsilon>0$ is arbitrary, this implies the desired inequalities. The lemma is proved.

Corollary 1. Suppose $\Lambda=\left\{\lambda_{k}, n_{k}\right\}_{k=1}^{\infty}$ has zero density. Then the indicator of the function $f(\lambda, \Lambda)$ coincides with the indicator of the function $F(\lambda, \Lambda)$.

Proof. By Lemma 3.2, we have

$$
\begin{aligned}
h_{f}(\lambda) & =\limsup _{t \rightarrow \infty} \ln |f(t \lambda, \Lambda)| / t \\
& \leq \limsup _{t \rightarrow \infty} \ln |F(t \lambda, \Lambda)| / t+\limsup _{t \rightarrow \infty} \alpha(t \lambda) / t=h_{F}(\lambda), \quad \lambda \in \mathbb{C} .
\end{aligned}
$$

Let $\left|\lambda_{0}\right|=1$. We choose numbers $0<t_{p} \rightarrow+\infty$ such that

$$
\lim _{p \rightarrow \infty} \ln \left|F\left(t_{p} \lambda_{0}, \Lambda\right)\right| / t_{p}=h_{F}\left(\lambda_{0}\right) .
$$

Since $\Lambda$ has zero density, Lemma 2.6 shows that

$$
\ln \left|F\left(t \lambda_{0}, \Lambda\right)\right| \geq \frac{t}{t_{p}} \ln \left|F\left(t_{p} \lambda_{0}, \Lambda\right)\right|-t \sum_{t_{p} / 2 \leq\left|\lambda_{k}\right|<3 t_{p} / 2} \frac{n_{k}}{\left|\lambda_{k}\right|}=\frac{t}{t_{p}} \ln \left|F\left(t_{p} \lambda_{0}, \Lambda\right)\right|-t \beta_{p},
$$

where $\beta_{p} \rightarrow 0, p \rightarrow \infty$, and $t_{p} / 2 \leq t<3 t_{p} / 2, p \geq 1$. By Lemma 3.1, $\mathcal{I}(\Lambda)$ is a $C^{0}$-set. Then $\mathcal{I}(\Lambda) \cap\left\{\lambda=r \lambda_{0}, r>0\right\}$ is an $E^{0}$-set. Thus, there exists a sequence $\left\{r_{p}\right\}_{p=p_{0}}^{\infty}$ such that $r_{p} \lambda_{0} \notin \mathcal{I}(\Lambda), t_{p} / 2 \leq r_{p}<3 t_{p} / 2, p \geq p_{0}$. Therefore, Lemma 3.2 and the above considerations imply

$$
\begin{aligned}
\frac{\ln \left|f\left(r_{p} \lambda_{0}, \Lambda\right)\right|}{r_{p}} & =\frac{\ln \left|F\left(r_{p} \lambda_{0}, \Lambda\right)\right|}{r_{p}}+\frac{\alpha\left(r_{p} \lambda_{0}\right)}{r_{p}} \\
& \geq \frac{\ln \left|F\left(t_{p} \lambda_{0}, \Lambda\right)\right|}{t_{p}}-\beta_{p}+\frac{\alpha\left(r_{p} \lambda_{0}\right)}{r_{p}} \rightarrow h_{F}\left(\lambda_{0}\right), \quad p \rightarrow \infty,
\end{aligned}
$$


showing that $h_{f}\left(\lambda_{0}\right) \geq h_{F}\left(\lambda_{0}\right)$. Due to the positive homogeneity of indicators, we have $h_{f}(\lambda) \geq h_{F}(\lambda), \in \mathbb{C}$. The corollary is proved.

It is easy to check that the type $\sigma$ of the function $F(\lambda, \Lambda)$ can be calculated by the formula $\sigma=\lim \sup _{|\lambda| \rightarrow \infty}|\mathcal{N}(|\lambda|, \Lambda)|$. Moreover, like for the function $f(\lambda, \Lambda)$ (see, e.g., [13. Section I.5, Theorem 5.6]), it coincides with the maximum of the indicator on the circle $|\lambda|=1$. Thus, Corollary 1 implies Cartwright's theorem (see [8] and [2, Section I.1, Theorem 1.1.8]).

Corollary 2. Suppose $\Lambda=\left\{\lambda_{k}, n_{k}\right\}_{k=1}^{\infty}$ has zero density. Then the type of the function $f(\lambda, \Lambda)$ coincides with the type of the function $F(\lambda, \Lambda)$.

Corollary 1 to Theorem 2.9 allows us to construct entire functions $f(\lambda, \Lambda)$ of exponential type with a given indicator $h_{f(\cdot, \Lambda)}\left(r e^{i \varphi}\right) \equiv r H(\varphi, K)$, where $K$ is an arbitrary compact convex set. We also see that $\Lambda \subseteq \Lambda_{\mathbb{Z}}$ is a regularly distributed set with the maximal possible angular density $\omega(\theta) \equiv \omega(\theta, K) / 2 \pi$.

Corollary 1 to Lemma 3.2 also makes it possible to construct entire functions $f(\lambda, \Lambda)$ with a given indicator $h_{f(\cdot, \Lambda)}\left(r e^{i \varphi}\right) \equiv r H(\varphi, K)$ for an arbitrary compact convex set $K$. Now $\Lambda$ is a set with the minimal possible angular density, i.e., with zero density. In this connection, we have the following proposition.

Corollary 3. Let $\mu_{k}, k \geq 1$, be a monotone nondecreasing sequence of positive numbers such that $\mu_{k} \rightarrow \infty, k \rightarrow \infty$, and $\lim _{k \rightarrow \infty} k / \mu_{k}=0$. We assume that $\sum_{k \geq 1}\left(\mu_{k}\right)^{-1}=+\infty$. Then for any compact convex set $K$, there exists a sequence $\varphi_{k} \in[0,2 \pi), k \geq 1$, such that the indicator of the function $f(\lambda, \Lambda)$ (where $\Lambda=\left\{\mu_{k} e^{i \varphi_{k}}, 1\right\}_{k=1}^{\infty}$ ) coincides with the support function $r H(\varphi, K)$ of the compact set $K\left(\lambda=r e^{i \varphi}\right)$.

Proof. Let $K$ be a compact convex set. By Corollary 1 to Lemma 3.2, it suffices to choose numbers $\varphi_{k}, k \geq 1$, such that the indicator of the function $F(\lambda, \Lambda)$ coincides with $r H(\varphi, K)$. First, suppose that $K$ has nonempty interior (i.e., is the closure of a convex domain) and $\widetilde{K}$ is the compact set complex conjugate to $K$. Since $\mu_{k} \rightarrow \infty$ and the series $\sum_{k \geq 1}\left(\mu_{k}\right)^{-1}$ diverges, there exists $k_{0}$ and a collection of numbers $\varphi_{l}, l=1, \ldots, k_{0}$, such that the point $z_{k_{0}}$, where

$$
z_{k}=\sum_{l=1}^{k} \frac{e^{-i \varphi_{l}}}{\mu_{l}},
$$

belongs to the boundary $\partial \widetilde{K}$ of the compact set $\widetilde{K}$. Here we may assume that $\widetilde{K}$ contains a disk of radius greater than $\left(\mu_{k}\right)^{-1}, k>k_{0}$. Then we can choose numbers $\varphi_{k}, k>k_{0}$, so that $z_{k} \in \partial \widetilde{K}$ and the transition from $z_{k}$ to $z_{k+1}$ along $\partial \widetilde{K}$ is carried out in the positive direction for all $k \geq k_{0}$.

By the same considerations as above, the sequence $\left\{z_{k}\right\}_{k=k_{0}}^{\infty}$ forms a dense set in $\partial \widetilde{K}$. Thus, by the definition of a support function, we obtain

$$
\begin{aligned}
\sup _{k \geq k_{0}} \operatorname{Re}\left(e^{i \varphi} z_{k}\right) & =\sup _{k \geq k_{0}} \operatorname{Re}\left(e^{-i \varphi} \bar{z}_{k}\right) \leq H(\varphi, K) \\
& \leq \limsup _{k \rightarrow \infty} \operatorname{Re}\left(e^{i \varphi} z_{k}\right)=\limsup _{r \rightarrow \infty} \frac{\left|F\left(r e^{i \varphi}, \Lambda\right)\right|}{r} .
\end{aligned}
$$

This gives us the desired statement in the case under consideration.

Next, let $K$ be a segment with ends $a$ and $b$. As above, we find a number $k_{0}$ and a collection of numbers $\varphi_{l}, l=1, \ldots, k_{0}$, such that $z_{k_{0}} \in \widetilde{K}$ and $|a-b| / 2>\left(\mu_{k}\right)^{-1}$, $k>k_{0}$. We choose $\varphi_{k}, k>k_{0}$, so that $z_{k} \in \tilde{K}, k>k_{0}$, and there exist two subsequences $z_{k(1, l)}, z_{k(2, l)}, l \geq 1$, converging to $\bar{a}$ and $\bar{b}$, respectively. Then the function $f(\lambda, \Lambda)$ has the desired property. 
Finally, suppose that $K=\{a\}$ consists of one point. We choose $\varphi_{k}, k \geq 1$, so that the sequence $z_{k}$ converges to $\bar{a}$ as $k \rightarrow \infty$. Then the indicator $F(\lambda, \Lambda)$ on the unit circle coincides with $\operatorname{Re}\left(e^{-i \varphi} a\right)=H(\varphi, K)$. The corollary is proved.

Theorem 3.3. Suppose $g$ is an entire function of exponential type, $\Lambda=\left\{\lambda_{k}, n_{k}\right\}_{k=1}^{\infty}$ is its zero set with multiplicities, and $\omega \in \Sigma_{0}$ is a function of general form. Assume that for all admissible $\varphi, \psi \notin \Phi(\omega)$ we have the inequality $\underline{n}_{0}(\Lambda(\varphi, \psi)) \geq \omega(\psi)-\omega(\varphi)$. Then $g=f_{1} f_{2}$, where $f_{1}, f_{2}$ are entire functions of exponential type, and the following is true:

1) the zero set $\widetilde{\Lambda} \subseteq \Lambda$ of the function $f_{1}$ has angular density $\omega$;

2) $\mathcal{N}(r, \widetilde{\Lambda}) \rightarrow 0, r \rightarrow+\infty$;

3) $f_{1}=f(\cdot, \widetilde{\Lambda})$ is of regular growth;

4) $h_{g}=h_{f_{1}}+h_{f_{2}}$.

Proof. In accordance with Corollary 2 to Theorem 2.9, there exists a regularly distributed set $\widetilde{\Lambda} \subseteq \Lambda$ with angular density $\omega$ such that $\mathcal{N}(r, \widetilde{\Lambda}) \rightarrow 0, r \rightarrow+\infty$. Then the Lindelöf theorem (see [1, Section I.11, Theorem 15]) shows that the function $f_{1}(\lambda)=f(\lambda, \widetilde{\Lambda})$, $\lambda \in \mathbb{C}$, is of exponential type and (as was noted at the beginning of the section) regular growth. We set $f_{2}=g / f_{1}$. Since the zero set of $f_{1}$ is a part of the zero set of $g$, we see that $f_{2}$ is an entire function. Hence, by Corollary 2 to Theorem 5 in Chapter III of the book [1] we have $h_{f_{2}} \equiv h_{g}(\lambda)-h_{f_{1}}(\lambda)$. In particular, it implies (see [1, I.18, Theorem 28]) that $f_{2}$ is of exponential type. The theorem is proved.

Lemma 3.4. Suppose that $\Lambda=\left\{\lambda_{k}, n_{k}\right\}_{k=1}^{\infty}$ and $f(\lambda, \Lambda)$ is an entire function of exponential type. Assume that $\Lambda$ has an angular density. Then $\omega_{\Lambda} \in \Sigma_{0}$.

Proof. By Lemma 2.8, for any $m \geq 1$ we have

$$
\mathcal{N}\left(2^{m+1}, \Lambda\right)-\mathcal{N}\left(2^{m}, \Lambda\right)=\bar{\mu} \ln 2+\varepsilon_{m}, \quad \varepsilon_{m} \rightarrow 0, \quad m \rightarrow \infty,
$$

where $\mu=\int_{0}^{2 \pi} e^{i \varphi} d \omega_{\Lambda}(\varphi)$. We assume that $\mu=|\mu| e^{i \vartheta} \neq 0$. Then

$$
\operatorname{Re}\left(e^{i \vartheta}\left(\mathcal{N}\left(2^{m+1}, \Lambda\right)-\mathcal{N}\left(2^{m}, \Lambda\right)\right)\right)=\operatorname{Re}\left(|\mu| \ln 2+e^{i \vartheta} \varepsilon_{m}\right) \geq|\mu| / 2, \quad m \geq m_{0} .
$$

Therefore, for all $m \geq m_{0}$ we have

$$
\begin{aligned}
\left|\mathcal{N}\left(2^{m+1}, \Lambda\right)\right| & \geq\left|\mathcal{N}\left(2^{m+1}, \Lambda\right)-\mathcal{N}\left(2^{m_{0}}, \Lambda\right)\right|-\left|N\left(2^{m_{0}}, \Lambda\right)\right| \\
& \geq \operatorname{Re}\left(e^{i \vartheta}\left(\mathcal{N}\left(2^{m+1}, \Lambda\right)-\mathcal{N}\left(2^{m_{0}}, \Lambda\right)\right)\right)-\left|\mathcal{N}\left(2^{m_{0}}, \Lambda\right)\right| \\
& \geq 2^{-1}\left(m+1-m_{0}\right)|\mu|-\left|\mathcal{N}\left(2^{m_{0}}, \Lambda\right)\right| .
\end{aligned}
$$

Thus, $\left|\mathcal{N}\left(2^{m}, \Lambda\right)\right| \rightarrow+\infty, m \rightarrow \infty$. On the other hand, since $f(\lambda, \Lambda)$ is of exponential type, the Lindelöf theorem (see [1, Section I.11, Theorem 15]) yields the existence of $c>0$ such that $|\mathcal{N}(r, \Lambda)| \leq c, r>0$. This contradiction means that $\mu=0$, i.e., $\omega_{\Lambda} \in \Sigma_{0}$. The lemma is proved.

Theorem 3.5. Suppose that $\Lambda=\left\{\lambda_{k}, n_{k}\right\}_{k=1}^{\infty}$ and $f(\lambda, \Lambda)$ is an entire function of exponential type. We assume that $\Lambda$ is of general form and has an angular density. Then $\omega_{\Lambda} \in \Sigma_{0}$, and $f(\cdot, \Lambda)=f_{1} f_{2}$, where $f_{1}, f_{2}$ are entire functions of exponential type, and the following is true:

1) the zero set $\widetilde{\Lambda} \subseteq \Lambda$ of $f_{1}$ has angular density $\omega=\omega_{\Lambda}$;

2) $\mathcal{N}(r, \tilde{\Lambda}) \rightarrow 0, r \rightarrow+\infty$;

3) $f_{1}=f(\cdot, \widetilde{\Lambda})$ is of regular growth;

4) $h_{f(\cdot, \Lambda)}=h_{f_{1}}+h_{f_{2}}$

5) the zero set $T=\Lambda \backslash \widetilde{\Lambda}$ of $f_{2}=f(\cdot, \Lambda)$ has zero density; 
6) there exists a $C^{0}$-set $\mathcal{R} \in \mathbb{C}$ such that

$$
\begin{aligned}
& \ln |f(\lambda, \Lambda)|=h(\lambda, \Lambda)+\operatorname{Re}(\mathcal{N}(|\lambda|, \Lambda) \lambda)+\alpha(\lambda), \quad \lambda \in \mathbb{C}, \\
& \lim _{\substack{|\lambda| \rightarrow \infty \\
\lambda \notin \mathcal{R}}} \alpha(\lambda) /|\lambda|=0, \quad \limsup _{|\lambda| \rightarrow \infty} \alpha(\lambda) /|\lambda|=0,
\end{aligned}
$$

where

$$
h\left(r e^{i \varphi}, \Lambda\right)=-r \int_{\varphi-2 \pi}^{\varphi}(\varphi-\theta) \sin (\varphi-\theta) d \omega_{\Lambda}(\theta)
$$

and we have $h_{f_{1}}(\lambda)=h(\lambda, \Lambda), \lambda \in \mathbb{C}$.

Proof. By Lemma 3.4, $\omega_{\Lambda} \in \Sigma_{0}$. Then $f(\cdot, \Lambda)=f_{1} f_{2}$ by Theorem 3.3, where $f_{1}, f_{2}$ are entire functions of exponential type, so that claims 1)-4) are valid. Statement 5) follows from the assumption of the theorem and 1). We prove 6). At the beginning of the section, it has been observed that for $f_{1}(\lambda)=f(\lambda, \widetilde{\Lambda})$ we have the representation

$$
\ln \left|f_{1}(\lambda)\right|=h_{f_{1}}(\lambda)+\alpha_{1}(\lambda), \quad \lambda \in \mathbb{C}, \lim _{\substack{|\lambda| \rightarrow \infty \\ \lambda \notin \mathcal{I}_{f_{1}}}} \alpha_{1}(\lambda) /|\lambda|=0,
$$

where $\mathcal{I}_{f_{1}}$ is a $C^{0}$-set. By statement 3) and the identity $\omega_{\widetilde{\Lambda}}=\omega_{\Lambda}$, we also have

$$
h_{f_{1}}\left(r e^{i \varphi}\right)=h\left(r e^{i \varphi}, \Lambda\right)=-r \int_{\varphi-2 \pi}^{\varphi}(\varphi-\theta) \sin (\varphi-\theta) d \omega_{\Lambda}(\theta) .
$$

Moreover (see [1, I.18, Theorem 28]), we obtain the relation

$$
\limsup _{|\lambda| \rightarrow \infty} \alpha_{1}(\lambda) /|\lambda|=0 \text {. }
$$

By Lemma 3.1 and 3.2, we have

$$
\begin{aligned}
\ln \left|f_{2}(\lambda)\right| & =\operatorname{Re}(\mathcal{N}(|\lambda|, T) \lambda)+\alpha_{2}(\lambda), \quad \lambda \in \mathbb{C}, \\
\lim _{\substack{|\lambda| \rightarrow \infty \\
\lambda \notin \mathcal{I}(T)}} \alpha_{2}(\lambda) /|\lambda| & =0, \quad \limsup _{|\lambda| \rightarrow \infty} \alpha_{2}(\lambda) /|\lambda|=0,
\end{aligned}
$$

where $\mathcal{I}(T)$ is a $C^{0}$-set. Using statement 3), we get

$$
\begin{aligned}
\operatorname{Re}(\mathcal{N}(|\lambda|, T) \lambda) & =\operatorname{Re}(\mathcal{N}(|\lambda|, \Lambda) \lambda)+\operatorname{Re}((\mathcal{N}(|\lambda|, T)-\mathcal{N}(|\lambda|, \Lambda)) \lambda) \\
& =\operatorname{Re}(\mathcal{N}(|\lambda|, \Lambda) \lambda)+\alpha_{3}(\lambda), \quad \lim _{|\lambda| \rightarrow \infty} \alpha_{3}(\lambda) /|\lambda|=0 .
\end{aligned}
$$

We set $\mathcal{R}=\mathcal{I}_{f_{1}} \cup \mathcal{I}(T)$ and $\alpha(\lambda)=\alpha_{1}(\lambda)+\alpha_{2}(\lambda)+\alpha_{3}(\lambda)$. Then (3.6)-(3.10) imply statement 6$)$. The theorem is proved.

Remarks. 1. Theorem 3.5 generalizes Levin's result for functions with a regularly distributed zero set (see [1, Section II.1, Theorem 2] for $\rho(r) \equiv 1$ ) to functions with a zero set that has an angular density.

2. Theorem 3.5 contains the main result of the paper [6] for $\rho=1$.

3. Theorem 3.5 involves the requirement that $\Lambda$ be of general form. We give an example showing that the theorem fails without this condition. Let $\Lambda=\Lambda^{-} \cup \Lambda^{+}$be the sequence from the example considered in the preceding section right after Corollary 3 to Theorem 2.9. It has the angular density $\omega=\omega(\cdot, K) \in \Sigma_{0}$, where $K$ is the segment $[-i \pi, i \pi]$ of the imaginary axis, and is not of general form. By (2.21) and Lindelöf's theorem cited above, the function $f(\lambda, \Lambda)$ is of exponential type. However, in that example it was shown that no regularly distributed set $\widetilde{\Lambda} \subseteq \Lambda$ with angular density $\omega$ can exist. Thus, the statement of Theorem 3.5 is false in this case. In particular, $f(\lambda, \Lambda)$ cannot be represented as a product of two functions one of which is of regular growth and the other vanishes on a set of zero density. 
Under some additional condition on $\Lambda$, Theorem 3.5 remains true without the assumption that $\Lambda$ is of general form. In this regard, we consider entire functions of exponential type in the class $A$ (see [1, Chapter V]), i.e., functions with a zero set $\Lambda=\left\{\lambda_{k}, n_{k}\right\}_{k=1}^{\infty}$ satisfying the condition $\sum_{k \geq 1}\left|\operatorname{Im}\left(n_{k} / \lambda_{k}\right)\right|<+\infty$.

Theorem 3.6. Suppose that $\Lambda=\left\{\lambda_{k}, n_{k}\right\}_{k=1}^{\infty}$ has an angular density and $f(\lambda, \Lambda)$ is a class $A$ entire function of exponential type. Then $\omega_{\Lambda} \in \Sigma_{0}$ and for some $\tau \geq 0$, we have $\omega_{\Lambda}=\omega(\cdot, K) / 2 \pi$, where $K=[-i \tau \pi, i \tau \pi]$. We also have $f(\cdot, \Lambda)=f_{1} f_{2}$, where $f_{1}, f_{2}$ are entire functions of exponential type, and for some $b_{1}, b_{2} \in \mathbb{R}$ the following is true:

1) the zero set $\tilde{\Lambda} \subseteq \Lambda$ of the function $f_{1}$ is regularly distributed and has the angular density $\omega=\omega_{\Lambda}$;

2) $\operatorname{Re} \mathcal{N}(r, \widetilde{\Lambda}) \rightarrow 0, r \rightarrow+\infty$;

3) $f_{1}=f(\cdot, \widetilde{\Lambda})$ is of regular growth and its indicator diagram is the segment $K+i b_{1}$ of the imaginary axis;

4) $h_{f(\cdot, \Lambda)}=h_{f_{1}}+h_{f_{2}}$;

5) the zero set $T=\Lambda \backslash \widetilde{\Lambda}$ of the function $f_{2}=f(\cdot, T)$ has zero density, and its indicator diagram is the segment $\left[a_{1}, a_{2}\right]+i b_{2}$ parallel to the real axis, where $a_{1}=$ $\lim \sup _{r \rightarrow+\infty} \operatorname{Re} \mathcal{N}(r, \Lambda)$ and $a_{2}=\liminf _{r \rightarrow+\infty} \operatorname{Re} \mathcal{N}(r, \Lambda)$

6) $\sum_{k \geq 1} \operatorname{Im}\left(n_{k} / \lambda_{k}\right)=b=b_{1}+b_{2}$;

7) there exists a $C^{0}$-set $\mathcal{R} \subset \mathbb{C}$ such that

$$
\begin{aligned}
\ln |f(\lambda, \Lambda)| & =\pi \tau|\operatorname{Im} \lambda|-b \operatorname{Im} \lambda+\operatorname{Re} \mathcal{N}(|\lambda|, T) \operatorname{Re} \lambda+\alpha(\lambda), \quad \lambda \in \mathbb{C}, \\
\lim _{\substack{|\lambda| \rightarrow \infty \\
\lambda \notin \mathcal{R}}} \alpha(\lambda) /|\lambda| & =0, \quad \limsup _{|\lambda| \rightarrow \infty} \alpha(\lambda) /|\lambda|=0 ;
\end{aligned}
$$

8) the indicator diagram of $f(\lambda, \Lambda)$ is the rectangle $\left[a_{1}, a_{2}\right]+[-i \tau \pi, i \tau \pi]+i b$.

Proof. By Lemma 3.4, we have $\omega_{\Lambda} \in \Sigma_{0}$. Then Lemma 2.10 shows that, for some $\tau \geq 0$, we have the identity $\omega_{\Lambda}=\omega(\cdot, K) / 2 \pi$, where $K=[-i \tau \pi, i \tau \pi]$, and there exists a set $T \subseteq \Lambda$ of zero density such that the limit $\lim _{r \rightarrow \infty} \mathcal{N}(r, \widetilde{\Lambda})$ exists, where $\widetilde{\Lambda}=\Lambda \backslash \Lambda$ and $\operatorname{Re} \mathcal{N}(r, \tilde{\Lambda}) \rightarrow 0, r \rightarrow \infty$. Thus, $\widetilde{\Lambda}$ is a regularly distributed set with angular density $\omega=\omega_{\Lambda}$.

Let $f_{1}(\lambda)=f(\lambda, \widetilde{\Lambda})$. Then statements 1$\left.), 2\right)$ are true. As in Theorem 3.5, we obtain

$$
\begin{gathered}
\ln \left|f_{1}(\lambda)\right|=h_{f_{1}}(\lambda)+\alpha_{1}(\lambda), \quad \lambda \in \mathbb{C}, \\
\limsup _{|\lambda| \rightarrow \infty} \alpha_{1}(\lambda) /|\lambda|=0, \lim _{\substack{|\lambda| \rightarrow \infty \\
\lambda \in \mathcal{I}_{f_{1}}}} \alpha_{1}(\lambda) /|\lambda|=0, \\
h_{f_{1}}\left(r e^{i \varphi}\right)=-b_{1} \operatorname{Im} \lambda-r \int_{\varphi-2 \pi}^{\varphi}(\varphi-\theta) \sin (\varphi-\theta) d \omega_{\Lambda}(\theta),
\end{gathered}
$$

where $\mathcal{I}_{f_{1}}$ is a $C^{0}$-set and

$$
b_{1}=\lim _{r \rightarrow \infty} \operatorname{Im} \mathcal{N}(r, \tilde{\Lambda})=\lim _{r \rightarrow \infty} \mathcal{N}(r, \tilde{\Lambda}) .
$$

From (3.12) and 1) we deduce statement 3). As in Theorem 3.3, the functions $f_{1}$ and $f_{2}=f(\cdot, T)$ are of exponential type, which yields 4$)$. Since $\omega_{\Lambda}=\omega(\cdot, K) / 2 \pi$, we have

$$
-r \int_{\varphi-2 \pi}^{\varphi}(\varphi-\theta) \sin (\varphi-\theta) d \omega_{\Lambda}(\theta)=r \pi \tau|\sin \varphi|
$$


By Lemmas 3.1 and 3.2, we have the representation

$$
\begin{aligned}
\ln \left|f_{2}(\lambda)\right| & =\operatorname{Re}(\mathcal{N}(|\lambda|, T) \lambda)+\alpha_{2}(\lambda), \quad \lambda \in \mathbb{C}, \\
\lim _{\substack{|\lambda| \rightarrow \infty \\
\lambda \notin \mathcal{I}(T)}} \alpha_{2}(\lambda) /|\lambda| & =0, \quad \limsup _{|\lambda| \rightarrow \infty} \alpha_{2}(\lambda) /|\lambda|=0,
\end{aligned}
$$

where $\mathcal{I}(T)$ is a $C^{0}$-set.

Let $b_{2}=\lim _{r \rightarrow \infty} \operatorname{Im} \mathcal{N}(r, \Lambda)$. Then

$$
b_{1}+b_{2}=\lim _{r \rightarrow \infty} \operatorname{Im}(\mathcal{N}(r, T)+\mathcal{N}(r, \tilde{\Lambda}))=\lim _{r \rightarrow \infty} \operatorname{Im} \mathcal{N}(r, \Lambda)=\sum_{k \geq 1} \operatorname{Im}\left(n_{k} / \kappa_{k}\right)=b,
$$

i.e., statement 6$)$ is true. By (3.14),

$$
\begin{aligned}
\ln \left|f_{2}(\lambda)\right| & =\operatorname{Re}(\mathcal{N}(|\lambda|, T) \lambda)+\alpha_{2}(\lambda) \\
& =\operatorname{Re} \mathcal{N}(|\lambda|, T) \operatorname{Re} \lambda-\operatorname{Im} \mathcal{N}(|\lambda|, T) \operatorname{Im} \lambda+\alpha_{2}(\lambda) \\
& =\operatorname{Re} \mathcal{N}(|\lambda|, T) \operatorname{Re} \lambda-b_{2} \operatorname{Im} \lambda+\alpha_{3}(\lambda)+\alpha_{2}(\lambda), \quad \lambda \in \mathbb{C}, \\
& \lim _{|\lambda| \rightarrow \infty} \alpha_{3}(\lambda) /|\lambda|=0 .
\end{aligned}
$$

Using statement 2) and Corollary 1 to Lemma 3.2, we get

$$
h_{f_{2}}(\lambda)=a_{1} \operatorname{Re} \lambda-b_{2} \operatorname{Im}, \operatorname{Re} \lambda>0, \quad h_{f_{2}}(\lambda)=a_{2} \operatorname{Re} \lambda-b_{2} \operatorname{Im} \lambda, \operatorname{Re} \lambda<0,
$$

which implies 5). Setting $\mathcal{R}=\mathcal{I}_{f_{1}} \cup \mathcal{I}(T)$ and $\alpha(\lambda)=\alpha_{1}(\lambda)+\alpha_{2}(\lambda)+\alpha_{3}(\lambda)$ and recalling (3.11)-(3.15), we get statement 7). Statement 8) follows from 3)-6). The theorem is proved.

\section{§4. Representation of ANALytic FunCtions}

Let $D$ be a convex domain in $\mathbb{C}$, and let $H(D)$ denote the space of functions analytic in $D$ with the topology of uniform convergence on compact subsets of $D$; finally, let $H^{*}(D)$ be the strong dual to $H(D)$.

Let $P_{D}$ be the space of entire exponential-type functions with conjugate diagrams lying in $D$. It is equipped with the standard topology of the inductive limit (see, e.g., [14, III.12.7]). The Laplace transform $f(\lambda)=\nu(\exp (\lambda z)), \nu \in H^{*}(D)$, establishes an isomorphism (see, e.g., [14, Section III.12, Theorems 12.3 and 12.13]) between the linear topological spaces $H^{*}(D)$ and $P_{D}$.

Suppose $\Lambda=\left\{\lambda_{k}, n_{k}\right\}_{k=1}^{\infty}$ and $\mathcal{E}(\Lambda)=\left\{z^{n} \exp \left(\lambda_{k} z\right)\right\}_{n=0, k=1}^{n_{k}-1, \infty}$. By the Hahn-Banach theorem, the system $\mathcal{E}(\Lambda)$ is not complete in the space $H(D)$ if and only if there exists a nonzero functional $\nu \in H^{*}(D)$ that vanishes at the elements of the system. Thus, the incompleteness of $\mathcal{E}(\Lambda)$ is equivalent to the existence of a function $f \in P_{D}$ (not identically zero) that vanishes at the points $\lambda_{k}$ with multiplicity at least $n_{k}, k \geq 1$. If the domain $D$ is empty, then for convenience we assume that any system $\mathcal{E}(\Lambda)$ is complete in $H(D)$.

Suppose $K$ is a compact convex set and $D(K)$ is the convex domain that is swept out while moving the compact $K$ inside $D$. The domain $D(K)$ is the union of all shifts $K+z$ of $K$ that lie in $D$. By the definition, we have $D(K) \subseteq D$. The domain $D(K)$ is empty if none of the shifts of $K$ lie in $D$. If $D(K) \neq \varnothing$, then, clearly, we have the representation $D(K)=D^{\prime}(K)+K$, where

$$
D^{\prime}(K)=\left\{z \in \mathbb{C}: \operatorname{Re}(z \lambda)<H_{D}(\lambda)-H_{K}(\lambda), \lambda \neq 0\right\},
$$

and $H_{L}(\lambda)=\sup _{z \in L} \operatorname{Re}(z \lambda)$ is the complex support function of a set $L$ whose restriction to the unit circle $\lambda=e^{i \varphi}$ coincides with the usual support function $H(\varphi, \widetilde{L})$ of the set $\widetilde{L}$ that is complex conjugate to $L$. 
Theorem 4.1. Suppose $K$ is a compact convex set, $D$ is a convex domain, and $\Lambda=$ $\left\{\lambda_{k}, n_{k}\right\}_{k=1}^{\infty}$. Assume that for every admissible $\varphi, \psi \notin \Psi(\widetilde{K})$, we have $\underline{n}_{0}(\Lambda(\varphi, \varphi)) \geq$ $(2 \pi)^{-1}(\omega(\psi, \tilde{K})-\omega(\varphi, \tilde{K}))$, where $\widetilde{K}$ is the compact set complex conjugate to $K$. Then the following statements are equivalent: 1) $\mathcal{E}(\Lambda)$ is complete in $H(D) ; 2) \mathcal{E}(\Lambda)$ is complete in $H(D(K))$.

Proof. 1) $\Rightarrow 2$ ). Suppose 1) is true. If $D(K)=\varnothing$, then 2) is also true. Assume that $D(K) \neq \varnothing$ and that $\mathcal{E}(\Lambda)$ is not complete in the space $H(D(K))$. Then there exists an entire function $f$ of exponential type that vanishes at the points $\lambda_{k}$ with multiplicity of at least $n_{k}, k \geq 1$, and such that its conjugate diagram lies in $D(K)$. Since $D(K) \subseteq D$, we have $f \in P_{D}$. This implies that $\mathcal{E}(\Lambda)$ is not complete in the space $H(D)$, which contradicts 1). Thus, $\mathcal{E}(\Lambda)$ is complete in $H(D(K))$.

$2) \Rightarrow 1$ ). Suppose 2) is true, but $\mathcal{E}(\Lambda)$ is not complete in $H(D)$. Then there exists $f \in P_{D}$ that vanishes at the points $\lambda_{k}$ with multiplicity of at least $n_{k}, k \geq 1$.

Using the assumption and Theorem 2.4, we see that there exists a sequence $\Lambda^{1} \subseteq \Lambda$ with angular density $\omega_{\Lambda^{1}}(\varphi)=(2 \pi)^{-1} \omega(\varphi, \tilde{K})$. Let $\Lambda^{2}=\Lambda^{1} \cup \Lambda_{\mathbb{Z}}$. For every admissible $\varphi, \psi$, we have $\underline{n}_{0}\left(\Lambda^{2}(\varphi, \psi)\right)=\underline{n}_{0}\left(\Lambda_{\mathbb{Z}}(\varphi, \psi)\right)=+\infty$. Therefore, by Theorem 2.9 , there exists a regularly distributed sequence $\widetilde{\Lambda}$ such that $\omega_{\tilde{\Lambda}}=\omega_{\Lambda^{1}}, \Lambda^{1} \subseteq \widetilde{\Lambda} \subseteq \Lambda^{2}$, and $\mathcal{N}(r, \tilde{\Lambda}) \rightarrow 0, r \rightarrow \infty$.

In the preceding section it was noted that the canonical function $f(\lambda, \widetilde{\Lambda})$ of the set $\widetilde{\Lambda}$ is of regular growth and

$$
h_{f(\cdot, \tilde{\Lambda})}\left(r e^{i \varphi}\right)=-r \int_{\varphi-2 \pi}^{\varphi}(\varphi-\theta) \sin (\varphi-\theta) d \omega_{\widetilde{\Lambda}}(\theta) .
$$

Moreover, there exists $z_{0} \in \mathbb{C}$ (see the remark to Lemma 2.7) such that

$$
H_{K}\left(r e^{i \varphi}\right)=r H(\varphi, \tilde{K})=r \operatorname{Re}\left(z_{0} e^{i \varphi}\right)+h_{f(\cdot, \Lambda)}\left(r e^{i \varphi}\right) .
$$

We set $T=\left\{\xi_{j}, m_{j}\right\}_{j=1}^{\infty}=\widetilde{\Lambda} \backslash \Lambda^{1}$. Then $T$ has zero density. The sequence $\widetilde{T}=-T \cup T$ (where $-T=\left\{-\xi_{j}, m_{j}\right\}_{j=1}^{\infty}$ ) also has zero density. Moreover, it is a regularly distributed set. Hence, the canonical function $f(\lambda, \widetilde{T})$ of the set $\widetilde{T}$ is of regular growth and has zero indicator $h_{f(\cdot, \widetilde{T})}=0$. We set $\tilde{f}(\lambda)=f(\lambda, \widetilde{T}) f(\lambda) / f(\lambda, \widetilde{\Lambda})$. Since the zero set of $f(\lambda, \widetilde{\Lambda})$ is a part of the zero set of the product $f(\lambda, \widetilde{T}) f(\lambda)$, it follows that $\tilde{f}$ is an entire function. Then by Corollary 2 to Theorem 5 in Chapter III of the book [1, we have the inequality

$$
h_{\widetilde{f}}(\lambda)=h_{f}(\lambda)+h_{f(\cdot, \widetilde{T})}(\lambda)-h_{f(\cdot, \widetilde{\Lambda})}(\lambda)=h_{f}(\lambda)-h_{f(\cdot, \widetilde{\Lambda})}(\lambda) .
$$

In particular, this implies that $\tilde{f}$ is of exponential type. Let $L$ and $P$ be the conjugate diagrams of the functions $f$ and $\tilde{f}$, respectively. Then, taking (4.1) into account, we have

$$
H_{P}(\lambda)=h_{\widetilde{f}}(\lambda)=h_{f}(\lambda)-h_{f(\cdot, \tilde{\Lambda})}(\lambda)=H_{L}(\lambda)-H_{K}(\lambda)+\operatorname{Re}\left(z_{0} \lambda\right) .
$$

It follows that $L=P+K-z_{0}$. Since $L \subset D$ (because $f \in P_{D}$ ), $L$ is the union of the shifts $K+z-z_{0}, z \in P$, of the compact set $K$, each of which lies in $D$. Thus, $L$ is a part of the domain $D(K)$ (in particular, $D(K) \neq \varnothing$ ). But then $f \in P_{D(K)}$. Therefore, the system $\mathcal{E}(\Lambda)$ is not complete in the space $H(D(K))$. This contradicts 2). Thus, our assumption is false, i.e., $\mathcal{E}(\Lambda)$ is complete in $H(D)$. The theorem is proved.

If the compact set $K$ coincides with the closure $\bar{D}$ of the domain $D$, then we obviously have $D(K)=\varnothing$. Therefore, Theorem 4.1 implies the next claim.

Corollary. Suppose $D$ is a bounded convex domain and $\Lambda=\left\{\lambda_{k}, n_{k}\right\}_{k=1}^{\infty}$. Assume that for every admissible $\varphi, \psi \notin \Psi(\widetilde{K})$ we have $\underline{n}_{0}(\Lambda(\varphi, \psi)) \geq(2 \pi)^{-1}(\omega(\psi, \widetilde{K})-\omega(\varphi, \widetilde{K}))$, 
where $\widetilde{K}$ is the compact set complex conjugate to $\bar{D}$. Then the system $\mathcal{E}(\Lambda)$ is complete in $H(D)$.

Under the assumptions of the corollary, each function $g \in H(D)$ can be represented as the limit (uniform on compact subsets of $D$ ) of linear combinations of elements of $\mathcal{E}(\Lambda)$. This representation can be "refined" for all functions $g \in H(\bar{D})$ (analytic in a neighborhood of $\bar{D}$ ) by expanding them in series of elements of $\mathcal{E}(\Lambda)$. This requires an additional condition on $\Lambda$ (in a sense, the points $\lambda_{k}$ should be "separated away" from each other).

We have the well-known Leont'ev criterion (see [2, Section IV.6, Theorem 4.6.4]) for the representation of functions $g \in H(\bar{D})$ as the series

$$
g(z)=\sum_{k=1}^{\infty} d_{k, 0} e^{\lambda_{k} z}, \quad z \in D,
$$

in the case where $\Lambda$ is the set of simple $\left(n_{k}=1\right)$ zeros of an entire function $f$ of exponential type whose conjugate diagram coincides with $\bar{D}$, and where the coefficients $d_{k, 0}$ are calculated via a system of functionals biorthogonal to $\mathcal{E}(\Lambda)$. This criterion consists of two conditions: the function $f$ must be of regular growth and following lower estimate for the absolute values of its derivatives at the points $\lambda_{k}$ must be fulfilled:

$$
\ln \left|f^{\prime}\left(\lambda_{k}\right)\right| \geq h_{f}\left(\lambda_{k}\right)-\varepsilon_{k}\left|\lambda_{k}\right|, \quad 0<\varepsilon_{k} \rightarrow 0, \quad k \rightarrow \infty .
$$

We provide conditions that ensure the representation (4.2), where the sequence $\Lambda=$ $\left\{\lambda_{k}, 1\right\}_{k=1}^{\infty}$ is arbitrary (it is not required to be a zero set of any entire function). These conditions are formulated solely in terms of geometrical characteristics of $\Lambda$ and $D$. For this, we need the "local" characteristic of the sequence $\Lambda$ introduced in 9 .

Let $\Lambda=\left\{\lambda_{k}, n_{k}\right\}_{k=1}^{\infty}$. Consider the function

$$
q_{\Lambda}(z, w, \delta)=\prod_{\lambda_{k} \in B(w, \delta|w|)}\left(\frac{z-\lambda_{k}}{3 \delta\left|\lambda_{k}\right|}\right)^{n_{k}} .
$$

In the case where the disk $B(w, \delta|w|)$ contains no $\lambda_{k}$, we set $q_{\Lambda}(z, w, \delta) \equiv 1$. The absolute value of the function $q_{\Lambda}(z, w, \delta)$ can be interpreted as a measure of concentration for the points $\lambda_{k} \in B(w, \delta|w|)$ near $z$. The quantity $\ln \left|q_{\Lambda}(z, w, \delta)\right| /|w|$ is similar to the logarithm of the geometric mean (the arithmetic mean of the logarithms) of the normalized distances from $\lambda_{k} \in B(w, \delta|w|)$ to $z$. If $\delta \in(0,1)$, then in the disk $B(w, \delta|w|)$ the absolute value of each factor in the definition of $q_{\Lambda}$ can be estimated from above by $2(3(1-\delta))^{-1}$. Then for $\delta \in(0,1 / 3)$, it does not exceed one. We set

$$
q_{\Lambda}^{m}(z, \delta)=\prod_{\substack{\lambda_{k} \in B\left(\lambda_{m}, \delta\left|\lambda_{m}\right|\right) \\ k \neq m}}\left(\frac{z-\lambda_{k}}{3 \delta\left|\lambda_{k}\right|}\right)^{n_{k}}, \quad S_{\Lambda}=\lim _{\delta \rightarrow 0} \liminf _{m \rightarrow \infty} \frac{\ln \left|q_{\Lambda}^{m}\left(\lambda_{m}, \delta\right)\right|}{\left|\lambda_{m}\right|} .
$$

The definition of $S_{\Lambda}$ implies the inequality $S_{\Lambda} \leq 0$ (see [9]).

Lemma 4.2. Let $\Lambda=\left\{\lambda_{k}, 1\right\}_{k=1}^{\infty}$ be the zero set of an entire function $f$ of exponential type and of regular growth. Assume that $S_{\Lambda}=0$. Then

$$
\ln \left|f^{\prime}\left(\lambda_{k}\right)\right| \geq h_{f}\left(\lambda_{k}\right)-\varepsilon_{k}\left|\lambda_{k}\right|, \quad 0<\varepsilon_{k} \rightarrow 0, \quad k \rightarrow \infty .
$$

Proof. Since $f$ is of regular growth, we have

$$
\ln |f(\lambda)|=h_{f}(\lambda)+\alpha(\lambda), \quad \lambda \in \mathbb{C}, \quad \lim _{\substack{|\lambda| \rightarrow \infty, \lambda \in \mathcal{I}_{f}}} \alpha(\lambda) /|\lambda|=0,
$$

where $\mathcal{I}_{f}$ is a $C^{0}$-set. We fix $\varepsilon>0$ and choose $R>0$ such that

$$
\alpha(\lambda) \geq-\varepsilon|\lambda|, \quad \lambda \in \mathcal{I}_{f}, \quad|\lambda| \geq R .
$$


Using the uniform continuity of the indicator on compact sets, we can also find $\delta_{0} \in$ $(0,1 / 3)$ satisfying

$$
\left|h_{f}(\lambda)-h_{f}(w)\right| \leq \varepsilon, \quad w \in B\left(\lambda, \delta_{0}\right), \quad|\lambda|=1 .
$$

In accordance with the assumption and the definition of $S_{\Lambda}$, we choose $\delta \in\left(0, \delta_{0}\right)$ and a number $k_{0}$ such that

$$
\left|\lambda_{k}\right| \geq 2 R, \quad \ln \left|q_{\Lambda}^{k}\left(\lambda_{k}, \delta\right)\right| \geq-\varepsilon\left|\lambda_{k}\right|, \quad k \geq k_{0} .
$$

Finally, since $\mathcal{I}_{f}$ is a $C^{0}$-set, we may assume that the following is fulfilled: for each $k \geq k_{0}$, the total sum of the exceptional disks in $\mathcal{I}_{f}$ that intersect $B\left(\lambda_{k}, \delta\left|\lambda_{k}\right|\right)$ does not exceed $\delta\left|\lambda_{k}\right| / 4$. Then by (4.4), (4.5), and (4.7), for any $k \geq k_{0}$ there exists $\alpha_{k} \in(1 / 2,1)$ such that

$$
\ln |f(\lambda)| \geq h_{f}(\lambda)-\varepsilon|\lambda|, \quad \lambda \in B\left(\lambda_{k}, \alpha_{k} \delta\left|\lambda_{k}\right|\right) .
$$

By the positive homogeneity of the indicator and (4.6), this implies

$$
\ln |f(\lambda)| \geq h_{f}\left(\lambda_{k}\right)-3 \varepsilon\left|\lambda_{k}\right|, \quad \lambda \in B\left(\lambda_{k}, \alpha_{k} \delta\left|\lambda_{k}\right|\right), \quad k \geq k_{0} .
$$

In accordance with the assumption, the function $\ln \left|f(\lambda) / q_{\Lambda}\left(\lambda, \lambda_{k}, \delta\right)\right|$ is harmonic in the disk $B\left(\lambda_{k}, \delta\left|\lambda_{k}\right|\right)$. Since $\delta<1 / 3$, the function $\ln \left|q_{\Lambda}\left(\lambda, \lambda_{k}, \delta\right)\right|$ is nonpositive in that disk. Thus, applying (4.8) and the minimum principle for harmonic functions, we obtain

$$
\ln \left|h_{k}\left(\lambda_{k}\right)\right| \geq h_{f}\left(\lambda_{k}\right)-3 \varepsilon\left|\lambda_{k}\right|, \quad k \geq k_{0} .
$$

Combined with (4.7), this implies

$$
\ln \left|f^{\prime}\left(\lambda_{k}\right)\right|=\ln \left|h_{k}\left(\lambda_{k}\right)\right|+\ln \left|q_{\Lambda}^{k}\left(\lambda_{k}, \delta\right)\right|-\ln \left(3 \delta\left|\lambda_{k}\right|\right) \geq h_{f}\left(\lambda_{k}\right)-5 \varepsilon\left|\lambda_{k}\right|, \quad k \geq k_{1} .
$$

The lemma is proved.

Remarks. 1. Estimate (4.3) implies that $S_{\Lambda}=0$. Here we do not demand the function $f$ to be of regular growth (see the proof of Corollary 4.2 in [15]).

2. The question as to whether estimate (4.3) implies that $f$ is of regular growth, remains open. Finding an answer to it is A. F. Leont'ev's problem .

3. Solely, the identity $S_{\Lambda}=0$ does not imply estimate (4.3) (see an example at the end of [16]).

Theorem 4.3. Suppose $D$ is a bounded convex domain and $\Lambda=\left\{\lambda_{k}, 1\right\}_{k=1}^{\infty}$. Assume that $S_{\Lambda}=0$ and that $\underline{n}_{0}(\Lambda(\varphi, \psi)) \geq(2 \pi)^{-1}(\omega(\psi, \widetilde{K})-\omega(\varphi, \widetilde{K}))$ for every admissible $\varphi, \psi \notin \Psi(\widetilde{K})$, where $\widetilde{K}$ is the compact set complex conjugate to $\bar{D}$. Then each function $g \in H(\bar{D})$ can be represented as a series of the form (4.2) converging uniformly on the compact subsets of $D$.

Proof. Since $D$ is a domain, $\omega(\psi, \widetilde{K}) \in \Sigma_{0}$ (see the remark to Lemma 2.7 ) and $\omega(\psi, \widetilde{K})$ is a function of general form. Then by Corollary 2 to Theorem 2.9, there exists a regularly distributed set $\widetilde{\Lambda} \subseteq \Lambda$ with the angular density $\omega=(2 \pi)^{-1} \omega(\cdot, \widetilde{K})$. At the beginning of $\S 3$, it was noted that in this case, the canonical function $f(\lambda, \tilde{\Lambda})$ is of regular growth and its indicator diagram (see the remark to Lemma 2.7) coincides with some shift $\widetilde{K}-z_{0}$ of the compact set $\tilde{K}$.

We set $f(\lambda)=f(\lambda, \widetilde{\Lambda}) e^{\lambda \bar{z}_{0}}$. Then the function $f$ is of regular growth and its conjugate diagram coincides with $\bar{D}$. By assumption, we have $S_{\Lambda}=0$. Since $\widetilde{\Lambda} \subseteq \Lambda$, the definition shows that all the factors of the function $q_{\tilde{\Lambda}}^{m}(z, \delta)$ are among the factors of $q_{\Lambda}^{m}(z, \delta)$. The absolute value of each of them does not exceed one, provided $\delta \in(0,1 / 3)$. This implies the inequality $S_{\widetilde{\Lambda}} \geq S_{\Lambda}=0$. As was noted above, we always have $S_{\widetilde{\Lambda}} \leq 0$. Thus, $S_{\widetilde{\Lambda}}=0$. 
Therefore, Lemma 4.2 is applicable. Using it, we obtain estimate (4.3). Hence, by [2, Theorem 4.6.4] each function $g \in H(\bar{D})$ can be represented in the form (4.2). The theorem is proved.

Remark. Suppose $\left\{K_{p}\right\}_{p=1}^{\infty}$ is a sequence of compact convex sets in the domain $D$ that strictly exhaust $D$, i.e., $K_{p} \subset \operatorname{int} K_{p+1}, p \geq 1$ (the symbol int means the interior of a set), and $D=\bigcup_{p=1}^{\infty} K_{p}$. For each $p \geq 1$, we introduce the following Banach space of sequences of complex numbers:

$$
Q_{p}=\left\{d=\left\{d_{k, n}\right\}:\|d\|_{p}=\sup _{\substack{k \geq 1, 0 \leq n<n_{k}}}\left|d_{k, n}\right| \exp H_{K_{p}}\left(\lambda_{k}\right)<\infty\right\}
$$

Suppose $Q(D)=\bigcap_{p \geq 1} Q_{p}$ is endowed with the topology of the projective limit. By [17, Lemma 2.3], the pointwise convergence of the series (4.2) in the domain $D$ implies that $d=\left\{d_{k, 0}\right\} \in Q(D)$. By Theorem 3.1 in the same paper [17] (an analog of Abel's theorem for power series), we also have

$$
\sum_{k=1}^{\infty}\left|d_{k, 0}\right| \max _{z \in K_{p}}\left|e^{\lambda_{k} z}\right| \leq C_{p}\|d\|_{p+2}, \quad p \geq 1,
$$

where $C_{p}>0$ is independent of $d=\left\{d_{k, 0}\right\} \in Q_{p}$. In particular, this means that the series (4.2) converges absolutely and uniformly on any compact subset of the domain $D$.

Suppose $D$ is a convex domain in $\mathbb{C}$ and $\Lambda=\left\{\lambda_{k}, n_{k}\right\}_{k=1}^{\infty}$ is such that $\mathcal{E}(\Lambda)$ is not complete in the space $H(D)$. Let $W(\Lambda, D)$ denote the closure of the linear span of the system $\mathcal{E}(\Lambda)$ in $H(D)$. Then $W(\Lambda, D)$ is a nontrivial closed subspace in $H(D)$ invariant with respect to the differentiation operator $(\Lambda$ is its multiple spectrum and $\mathcal{E}(\Lambda)$ is the set of eigenfunctions and associated functions in $W(\Lambda, D))$.

By construction, $\mathcal{E}(\Lambda)$ is complete in $W(\Lambda, D)$. In this case, $W(\Lambda, D)$ is said to admit spectral synthesis. In the same way we can obtain any nontrivial closed subspace in $H(D)$ invariant with respect to differentiation and admitting spectral synthesis. An example of such a space is the set of solutions of the convolution equation $\mu(g(z+w)) \equiv 0(\mu \neq 0$ is a continuous linear functional on $H(D)$ ), because by Theorem 6.1 in [18] it admits spectral synthesis. Special cases of the convolution equation are linear differential, difference, differential-difference equations with constant coefficients of finite and infinite orders as well as certain types of integral equations.

Each function $\in W(\Lambda, D)$ is the limit of a sequence of linear combinations of elements of $\mathcal{E}(\Lambda)$. Naturally, the problem arises of representing $g$ as a series of elements of $\mathcal{E}(\Lambda)$. The first result in this direction was L. Euler's fundamental principle. It states that each solution of a linear homogeneous differential equation with constant coefficients is a linear combination of elementary solutions belonging to $\mathcal{E}(\Lambda)$. In this regard, the problem of representing functions in $W(\Lambda, D)$ as series

$$
\sum_{k=1, n=0}^{\infty, n_{k}-1} d_{k, n} z^{n} \exp \left(\lambda_{k} z\right)
$$

is called the fundamental principle problem in an invariant subspace. Via the Laplace transformation, this problem reduces to the problem of multiple interpolation in the space of entire functions of exponential type. These two problems have initially been studied independently, and they have a rich history. The main stages of that history are presented in [19] and [9]. The paper [9] provides solutions of the fundamental principle problem and of the interpolation problem for an arbitrary convex domain $D \subset \mathbb{C}$ under the condition that $n_{k} /\left|\lambda_{k}\right| \rightarrow 0, k \rightarrow \infty$. In the paper [10, this condition was lifted for a bounded domain. Hence, the paper [10] provides a criterion of the fundamental 
principle for the invariant subspace $W(\Lambda, D)$ in a bounded convex domain $D$. This criterion consists of two conditions: $S_{\Lambda}=0$ (local) and $\Lambda$ is compatible with $D$ (global). The latter means that $\Lambda$ is a part of the zero set of an entire function of exponential type and of regular growth whose conjugate diagram coincides with $\bar{D}$.

Theorem 2.9 provides a criterion for the fundamental principle in the case of the invariant subspace $W(\Lambda, D)$ in a bounded convex domain $D$, formulated solely in terms of geometrical characteristics of the sequence $\Lambda$ and the domain $D$.

We define an operator $\mathcal{L}$ on the space $Q(D)$ as follows. We associate each sequence $d=\left\{d_{k, n}\right\} \in Q(D)$ with the sum of the series (4.9) uniformly convergent on the compact subsets of $D$.

Theorem 4.4. Suppose that $D$ is a bounded convex domain and that $\Lambda=\left\{\lambda_{k}, n_{k}\right\}_{k=1}^{\infty}$, $\left|\lambda_{k}\right|+\infty$, is such that $\mathcal{E}(\Lambda)$ is not complete. The following statements are equivalent:

1) each function in $W(\Lambda, D)$ can be represented as a series of the form (4.9) uniformly convergent on the compact subsets of $D$;

2 ) the operator $\mathcal{L}$ is an isomorphism between the linear topological spaces $Q(D)$ and $W(\Lambda, D)$;

3) $S_{\Lambda}=0$ and for every admissible $\varphi, \psi \notin \Psi(\widetilde{K})$ we have

$$
\bar{n}_{0}(\Lambda(\varphi, \psi)) \leq(2 \pi)^{-1}(\omega(\psi, \tilde{K})-\omega(\varphi, \tilde{K})),
$$

where $\widetilde{K}$ is the compact set complex conjugate to $\bar{D}$.

Proof. The equivalence of 1) and 2) was proved in Theorem 3 in [10. Suppose 1) is true. Then by that theorem, we have $S_{\Lambda}=0$ and there exists an entire function $f$ of exponential type and of regular growth such that $h_{f}(\lambda)=H_{D}(\lambda), \lambda \in \mathbb{C}$ (i.e., the conjugate diagram of the function $f$ coincides with $\bar{D}$ ). Also, $\Lambda$ is a part of the zero set $\widetilde{\Lambda}$ of the function $f$. In this case (as was noted in the preceding section), the angular density $\omega_{\widetilde{\Lambda}}(\varphi)$ coincides with $\omega(\varphi, \widetilde{K}) / 2 \pi$. Since $\Lambda \subseteq \widetilde{\Lambda}$, we have

$$
\bar{n}_{0}(\Lambda(\varphi, \psi)) \leq \bar{n}_{0}(\widetilde{\Lambda}(\varphi, \psi))=n(\widetilde{\Lambda}(\varphi, \psi))=(2 \pi)^{-1}(\omega(\psi, \widetilde{K})-\omega(\varphi, \widetilde{K}))
$$

which implies statement 3 ).

Next, suppose 3 ) is fulfilled. By Corollary 3 to Theorem 2.9, there exists a regularly distributed set $\widetilde{\Lambda}$ with the angular density $\omega_{\widetilde{\Lambda}}(\varphi)=\omega(\varphi, \widetilde{K}) / 2 \pi$. As in Theorem 4.3, there exists a function $f$ of regular growth such that its conjugate diagram coincides with $\bar{D}$. Then 1) is true by Theorem 3 in [10]. The theorem is proved.

\section{REFERENCES}

[1] B. Ya. Levin, Distribution of zeros on entire functions, Gosudarstv. Izdat. Tehn.-Teor. Lit., Moscow, 1956. MR0087740 (19,402c) English transl., Transl. Math. Monogr., vol. 5, Amer. Math. Soc., Providence, RI, 1980. MR0156975 (28:217)

[2] A. F. Leont'ev, Exponential series, Nauka, Moscow, 1976. (Russian) MR0584943

[3] G. Polya, Untersuchungen uber Lücken und Singularitaten von Potenzreihen, Math. Z. 29 (1929), no. 1, 549-640. MR 1545027

[4] A. A. Kondratyuk, Entire functions with positive zeros that have a finite maximal density, Teor. Funkcii Funkcional. Anal. i Prilozhen. (Kharkov) 7 (1968), 37-52. (Russian) MR0277717

[5] G. L. Luntz, A certan theorem that is connected with the grawth of entire functions of entire order, Izv. Akad. Nauk Armjan. SSR. Ser. Mat. 5 (1970), no. 4, 358-370. (Russian) MR0288264

[6] G. N. Shilova, A theorem on the divisors of finite-order entire functions, Mat. Zametki 48 (1990), no. 2, 128-136; English transl., Math. Notes 48 (1990), no. 1-2, 799-804. MR1076943

[7] A. I. Abdulagimov and A. S. Krovosheyev, Properly distributed subsequence on the line, Ufim. Mat. Zh. 7 (2015), no. 1, 3-12; English transl., Ufa Math. J. 7 (2015), no. 1, 3-12. MR3430737

[8] M. L. Cartwright, On integral functions of integral order, Proc. London Math. Soc. 33 (1931), no. 1, 209-224. MR 1576826 
[9] A. S. Krivosheev, The fundamental principle for invariant subspaces in convex domains, Izv. Ross. Akad. Nauk Ser. Mat. 68 (2004), no. 2, 71-136; English transl., Izv. Math. 68 (2004), no. 2, 291-353. MR2058001

[10] O. A. Krivosheeva and A. S. Krivosheev, Criterion for the fudamental principle to hold for invariant subspaces in bounded convex domains in the complex plane, Functsional. Anal. i Prilozhen. 46 (2012), no. 4, 14-30; English transl., Funct. Anal. Appl. 46 (2012), no. 4, 249-261. MR3075093

[11] _ A closedness of set of Dirichlet series sums, Ufim. Mat. Zh. 5 (2013), no. 3, 96-120; English transl., Ufa Math. J. 5 (2013), no. 3, 94-117. MR3430790

[12] A. A. Kondratyuk, Entire functions with finite maximal density of zeros, Teor. Funkcii Funkcional. Anal. i Prilozhen. (Kharkov) 10 (1970), 57-70. (Russian) MR0298000

[13] A. F. Leont'ev, Entire functions. Series of exponentials, Nauka, Moscow, 1983. (Russian) MR0753827 (86j:30005)

[14] V. V. Napalkov, Convolution equations in multidimensional spaces, Nauka, Moscow, 1982. (Russian) MR 0678923

[15] O. A. Krivosheeva, Singular points of the sum of series of exponential monomials on the boundary of the convergence domain, Algebra i Analiz 23 (2011), no. 2, 162-205; English transl., St. Peterburg Math. J. 23 (2012), no. 2, 321-350. MR2841675

[16] O. A. Krivosheeva and A. S. Krovosheev, Singular points of the sum of a Dirichlet series on the convergence line, Functsional. Anal. i Prilozhen. 49 (2015), no. 2. 54-69; English transl., Funct. Anal. Appl. 49 (2015), no. 2, 122-134. MR.3374903

[17] O. A. Krivosheeva, The convergence domain for series of exponential monomials, Ufim. Mat. Zh. 3 (2011), no. 2, 43-56; English transl., Ufa Math. J. 3 (2011), no. 2, 42-55. MR3428992

[18] I. F. Krasičkov-Ternovskiŭ, Invariant subspaces of analytic functions. II. Spectral synthesis on convex domains, Mat. Sb. 88 (1972), no. 1, 3-30. (Russian) MR.0422636

[19] A. A. Gol'dberg, B. Ya. Levin, and I. V. Ostrovskiı̆, Entire and meromorphic functions. Itogi Nauki i Tekhniki, Sovremen. Probl. Math., vol. 256, VINITI, Moscow, 1991, pp. 5-186. MR.1155417

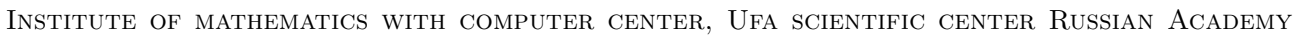
of Sciences, Chernyshevskil str. 112, 450048 Ufa, Russia

E-mail address: buffonishe@mail.ru

Bashkir State University, Zaki Validi str. 32, 450076 Ufa, Russia

E-mail address: kriolesya2006@yandex.ru

Received 25/JUN/2015

Translated by N. N. OSIPOV 